\title{
EFFECTIVE CIRCLE COUNT FOR APOLLONIAN PACKINGS AND CLOSED HOROSPHERES
}

\author{
MIN LEE AND HEE OH
}

\begin{abstract}
The main result of this paper is an effective count for Apollonian circle packings that are either bounded or contain two parallel lines. We obtain this by proving an effective equidistribution of closed horospheres in the unit tangent bundle of a geometrically finite hyperbolic 3-manifold, whose fundamental group has critical exponent bigger than 1. We also discuss applications to Affine sieves. Analogous results for surfaces are treated as well.
\end{abstract}

\section{INTRODUCTION}

1.1. Apollonian circle packings. An Apollonian circle packing is an ancient Greek construction which is made by repeatedly inscribing circles into the triangular interstices of four mutually tangent circles in the plane. In recent years, there have been many new and exciting developments in the study of Apollonian circle packings; for instance, see [15], [16], [32], [6], [20], [3], [4], [26], [27], [25], etc.

The main goal of this paper is to obtain an effective version of the counting theorem for circles in an Apollonian packing with bounded curvature.

Let $\mathcal{P}$ be an Apollonian circle packing, that is either bounded or lies between two parallel lines (i.e., congruent to the packing in Figure 2). For $T>0$ and $\mathcal{P}$ bounded, we define the circle counting function as follows:

$$
N_{T}(\mathcal{P}):=\#\{C \in \mathcal{P}: \operatorname{Curv}(C)<T\}
$$

where $\operatorname{Curv}(C)$ denotes the curvature of $C$, i.e., the reciprocal of the radius of $C$. For $\mathcal{P}$ unbounded between two parallel lines, we adjust the definition of $N_{T}(\mathcal{P})$ to count circles only in a fixed period.

The main term in the asymptotic for $N_{T}(\mathcal{P})$ will be described in terms of the residual set of $\mathcal{P}$ (=the closure of the union of all circles in $\mathcal{P}$ ), denoted by $\operatorname{Res}(\mathcal{P})$. We denote by $\alpha$ the Hausdorff dimension of $\operatorname{Res}(\mathcal{P}) ; \alpha$ is independent of $\mathcal{P}$ and known to be approximately

The authors are respectively supported in parts by Simons Fellowship and by NSF Grant \#1068094. 
1.30568(8) [24]. Let $\mathcal{H}^{\alpha}(\operatorname{Res}(\mathcal{P}))$ be the $\alpha$-dimensional Hausdorff measure of $\operatorname{Res}(\mathcal{P})$ for bounded $\mathcal{P}$. For $\mathcal{P}$ between two parallel lines, we let $\mathcal{H}^{\alpha}(\operatorname{Res}(\mathcal{P}))$ be the measure of $\operatorname{Res}(\mathcal{P})$ in a fixed period.

The error term in our asymptotic formula depends directly on the $L^{2}$ spectral gap of the complete hyperbolic 3 manifold whose fundamental group is the symmetry group of $\mathcal{P}$. The group $\mathrm{PSL}_{2}(\mathbb{C})$ acts on the extended plane by linear fractional transformations. Set

$$
\mathcal{A}_{\mathcal{P}}:=\left\{g \in \mathrm{PSL}_{2}(\mathbb{C}): g(\mathcal{P})=\mathcal{P}\right\} .
$$

It is known that $\mathcal{A}_{P}$ is a geometrically finite discrete subgroup of $\mathrm{PSL}_{2}(\mathbb{C})$ with critical exponent equal to $\alpha$ (cf. [20]). The fact $\alpha$ is strictly bigger than 1 yields that $\alpha(2-\alpha)$ is the smallest eigenvalue of the Laplacian $\Delta$ on the $L^{2}$-spectrum of the hyperbolic manifold $\mathcal{A}_{\mathcal{P}} \backslash \mathbb{H}^{3}$ by Sullivan [36] and is also isolated by Lax and Phillips [22]. Hence there exists $1<s_{1}<\alpha$ such that there is no eigenvalue of $\Delta$ in $L^{2}\left(\mathcal{A}_{\mathcal{P}} \backslash \mathbb{H}^{3}\right)$ between $\alpha(2-\alpha)$ and $s_{1}\left(2-s_{1}\right)$. Since all $\mathcal{A}_{\mathcal{P}}$ 's are conjugate to each other by elements of $\mathrm{PSL}_{2}(\mathbb{C}), s_{1}$ is independent of $\mathcal{P}$.

Our effective counting result, which is a special case of our more general theorem (Theorem 1.8), can be stated as follows:

Theorem 1.1. As $T \rightarrow \infty$,

$$
N_{T}(\mathcal{P})=c_{A} \cdot \mathcal{H}^{\alpha}(\operatorname{Res}(\mathcal{P})) \cdot T^{\alpha}+O\left(T^{\alpha-\frac{2\left(\alpha-s_{1}\right)}{63}}\right)
$$

where $c_{A}>0$ is a constant independent of $\mathcal{P}$.

Remark 1.2. (1) In [20], the asymptotic $N_{T}(\mathcal{P}) \sim c_{\mathcal{P}} \cdot T^{\alpha}$ was obtained with less clear interpretation of the constant $c_{\mathcal{P}}$.

(2) A similar type of asymptotic formula was obtained in [27] for all Apollonian packings (whether bounded or not) by counting circles in a bounded region, but with no error term.

(3) There are several different ways of understanding the constant $c_{A} \cdot \mathcal{H}^{\alpha}(\operatorname{Res}(\mathcal{P}))$ in front of the main term, due to different approaches to the counting problem. One description is given in our paper (see (8.8)). The aforementioned paper [27] gives another expression as well.

(4) An Apollonian packing $\mathcal{P}$ is called integral if the curvatures of all circles in $\mathcal{P}$ are integers. Any integral Apollonian packing is known to be either bounded or lies between two parallel lines. Therefore Theorem 1.1 applies to all integral Apollonian packings. 


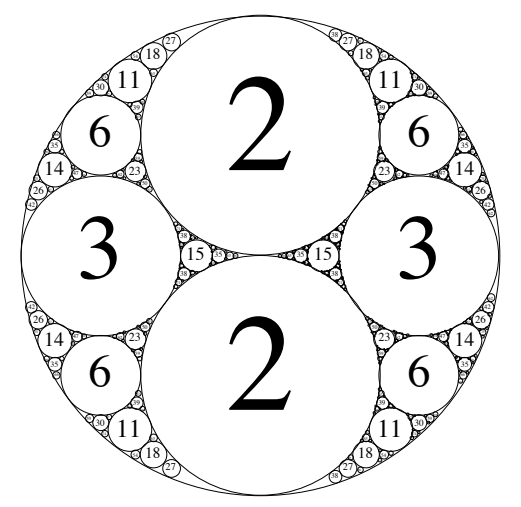

Figure 1. A bounded Apollonian circle packing.

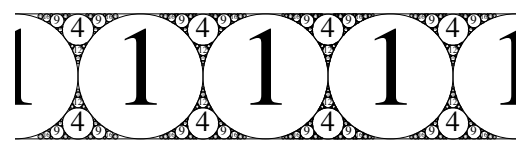

Figure 2. An unbounded Apollonian circle packing bounded by two parallel line.

Based on the Descartes circle theorem [9], the approach in [20] was to relate the circle counting problem with the equidistribution of closed horospheres in the unit tangent bundle of the hyperbolic manifold $\mathcal{A}_{\mathcal{P}} \backslash \mathbb{H}^{3}$.

The new achievement of this paper is an effective equidistribution of closed horospheres (Theorem 1.3). Besides its application to counting problems, such equidistribution result is of independent interest in homogeneous dynamics.

1.2. Effective equidistribution of closed horospheres. We obtain an effective equidistribution for closed horospheres in the unit tangent bundle of hyperbolic $n$-manifolds for $n=2$ or 3 . Consider the upper half space $\mathbb{H}^{n}=\left\{(x, y): x \in \mathbb{R}^{n-1}, y>0\right\}$ with the metric given by $d s^{2}=\frac{\sum_{i=1}^{n-1} d x_{i}^{2}+d y^{2}}{y^{2}}$ and let $G=\operatorname{Isom}^{+}\left(\mathbb{H}^{n}\right)$ denote the group of orientation preserving isometries of $\mathbb{H}^{n}$. That is, $G=\mathrm{PSL}_{2}(\mathbb{R})$ for $n=2$ and $G=\mathrm{PSL}_{2}(\mathbb{C})$ for $n=3$.

Let $\Gamma<G$ be a torsion-free discrete subgroup, which is not virtually abelian. We assume that $\Gamma$ is geometrically finite, that is, it admits a finite sided fundamental domain in $\mathbb{H}^{n}$. The limit set $\Lambda(\Gamma)$ is the subset 
of the boundary $\partial\left(\mathbb{H}^{n}\right)=\mathbb{R}^{n} \cup\{\infty\}$ consisting of all accumulation points in an orbit $\Gamma(z), z \in \mathbb{H}^{n}$. We denote by $0<\delta \leq n-1$ the critical exponent of $\Gamma$; it is equal to the Hausdorff dimension of $\Lambda(\Gamma)$ [37.

For $G=\mathrm{PSL}_{2}(\mathbb{R})$, set $K:=\operatorname{PSO}(2)$, and for $G=\mathrm{PSL}_{2}(\mathbb{C})$, set $K:=\operatorname{PSU}(2)$. In both cases, set

$$
A:=\left\{a_{y}:=\left(\begin{array}{cc}
\sqrt{y} & 0 \\
0 & \frac{1}{\sqrt{y}}
\end{array}\right): y>0\right\},
$$

and let $M$ be the centralizer of $A$ in $K$.

The hyperbolic manifold $\Gamma \backslash \mathbb{H}^{n}$ and its unit tangent bundle $\mathrm{T}^{1}\left(\Gamma \backslash \mathbb{H}^{n}\right)$ can be identified with the double quotient spaces $\Gamma \backslash G / K$ and $\Gamma \backslash G / M$ respectively. Accordingly, functions on $\Gamma \backslash \mathbb{H}^{n}$ (resp. $\mathrm{T}^{1}\left(\Gamma \backslash \mathbb{H}^{n}\right)$ ) can be considered as right $K$-invariant (resp. $M$-invariant) functions on $\Gamma \backslash G$. Since $a_{y}$ commutes with $M, a_{y}$ acts on $\Gamma \backslash G / M$ by the multiplication from the right and this action corresponds to the geodesic flow in the unit tangent bundle.

Set $N=\left\{g \in G: a_{y}^{-1} g a_{y} \rightarrow e\right.$ as $\left.y \rightarrow \infty\right\}$; the contracting horospherical subgroup under the action of $a_{y}$. Setting

$$
n_{x}:=\left(\begin{array}{cc}
1 & x \\
0 & 1
\end{array}\right)
$$

we have $N=\left\{n_{x}: x \in \mathbb{R}\right\}$ for $G=\mathrm{PSL}_{2}(\mathbb{R})$, and $N=\left\{n_{x}: x \in \mathbb{C}\right\}$ for $G=\mathrm{PSL}_{2}(\mathbb{C})$. Even though there is no action of $N$ on $\Gamma \backslash G / M$, the $N$-orbits $\{[g] N:=\Gamma \backslash \Gamma g M N / M: g \in G\}$ are well-defined since $N$ is normalized by $M$; these orbits give rise to the stable horospherical foliation of $\mathrm{T}^{1}\left(\Gamma \backslash \mathbb{H}^{n}\right)$.

In the rest of the introduction, we assume that $(n-1) / 2<\delta<n-1$ and that $\Gamma \backslash \Gamma N$ is closed in $\Gamma \backslash G$. In particular, $\Gamma$ has infinite covolume in $G$. By the torsion-free assumption on $\Gamma, \Gamma \cap N M=\Gamma \cap N$ and we can identify $\Gamma \backslash \Gamma N M / M$ with $(\Gamma \cap N) \backslash N$. Note that the quotient $(\Gamma \cap N) \backslash N$ can be naturally identified with $(\mathbb{R} / \mathbb{Z})^{k} \times \mathbb{R}^{n-1-k}$ where $0 \leq k \leq n-1$ denotes the rank of the free abelian subgroup $\Gamma \cap N$.

1.3. Equidistribution in spectral terms. We describe the effective equidistribution of $\Gamma \backslash \Gamma N a_{y}$ as $y \rightarrow 0$ in $\mathrm{T}^{1}\left(\Gamma \backslash \mathbb{H}^{n}\right)$ in terms of the $M$-invariant spectrum of $L^{2}(\Gamma \backslash G)$ for a Casimir element of $G$.

By Lax and Phillips [22] and Sullivan [36], the Laplacian $\Delta$ on $L^{2}\left(\Gamma \backslash \mathbb{H}^{n}\right)$ has only finitely many eigenvalues

$$
0<\alpha_{0}=\delta(n-1-\delta)<\alpha_{1} \leq \cdots \leq \alpha_{k}<\frac{(n-1)^{2}}{4}
$$


lying below the continuous spectrum $\left[\frac{(n-1)^{2}}{4}, \infty\right)$. The existence of a point eigenvalue is the precise reason that our main theorem requires the condition $\delta>(n-1) / 2$. Writing $\alpha_{1}=s_{1}\left(n-1-s_{1}\right)$, any positive number

$$
0<\mathbf{s}_{\Gamma}<\delta-s_{1}
$$

will be referred to as a spectral gap of $\Gamma$.

Let $\mathcal{C}$ denote a Casimir element of $\operatorname{Lie}(G)_{\mathbb{C}}$, which we normalize so that it acts on $K$-invariant smooth functions as the negative Laplacian $-\Delta$. Then $L^{2}(\Gamma \backslash G)$ contains the unique irreducible infinite dimensional subrepresentation $V$ (a complementary series representation) on which $\mathcal{C}$ acts by the scalar $\delta(\delta-n+1)$.

Let $\hat{K}$ denote the unitary dual of $K$, that is, the equivalence classes of all irreducible unitary representations of $K$. For $n=2, \hat{K}$ can be parametrized by $\mathbb{Z}$ so that $\ell \in \hat{K}$ corresponds to the one-dimensional space $V_{\ell}$ on which $k_{\theta}=\left(\begin{array}{cc}\cos \theta & \sin \theta \\ -\sin \theta & \cos \theta\end{array}\right)$ acts by $e^{2 \ell i \theta}$. For $n=3, \hat{K}$ can be parametrized by $\mathbb{Z}_{\geq 0}$ so that $\ell \in \hat{K}$ corresponds to the irreducible $2 \ell+1$ dimensional representation $V_{\ell}$.

As a $K$-representation, $V$ is decomposed into the orthogonal sum $\oplus_{\ell \in \hat{K}} V_{\ell}$ with the subspace $V_{\ell}^{M}$ of $M$-invariant vectors being one dimensional. Let $\phi_{\ell} \in C^{\infty}(\Gamma \backslash G) \cap L^{2}(\Gamma \backslash G)$ be a unit vector in $V_{\ell}^{M}$ for each $\ell \in \hat{K}$. We show that there exists $c_{n}(\ell) \neq 0$ such that for all $y>0$,

$$
\int_{n_{x} \in(N \cap \Gamma) \backslash N} \phi_{\ell}\left(n_{x} a_{y}\right) d x=c_{n}(\ell) \cdot y^{n-1-\delta} .
$$

The inner product $\left\langle\psi_{1}, \psi_{2}\right\rangle$ in $L^{2}(\Gamma \backslash G)$ is given by

$$
\left\langle\psi_{1}, \psi_{2}\right\rangle=\int_{\Gamma \backslash G} \psi_{1}(g) \overline{\psi_{2}(g)} d g
$$

where $d g$ denotes a $G$-invariant measure on $\Gamma \backslash G$.

The following is our main theorem on the effective equidistribution:

Theorem 1.3. Let $n=2$ or 3. Let $(n-1) / 2<\delta<n-1$. For any $\psi \in C_{c}^{\infty}(\Gamma \backslash G)^{M}$, as $y \rightarrow 0$,

$$
\begin{aligned}
\int_{(N \cap \Gamma) \backslash N} & \psi\left(n_{x} a_{y}\right) d x \\
& =\sum_{\ell \in \hat{K}} c_{n}(\ell) \cdot\left\langle\psi, \phi_{\ell}\right\rangle \cdot y^{n-1-\delta}+O\left(\mathcal{S}_{2 n-1}(\psi) \cdot y^{(n-1-\delta)+\frac{2 \mathbf{s}_{\Gamma}}{2 n+1}}\right)
\end{aligned}
$$


where $\mathcal{S}_{2 n-1}(\psi)$ denotes the $L^{2}$-Sobolev norm of $\psi$ of order $2 n-1$. Moreover

$$
c_{n}(\ell)=O\left((|\ell|+1)^{(n-2) / 2}\right) \quad \text { and } \sum_{\ell \in \hat{K}}\left|c_{n}(\ell)\left\langle\psi, \phi_{\ell}\right\rangle\right|=O\left(\mathcal{S}_{2}(\psi)\right) .
$$

Remark 1.4. (1) When $\Gamma$ is a lattice in $G$, i.e., when $\delta=n-1$, an effective equidistribution for expanding closed horospheres is well known, via the mixing of the geodesic flow and the thickening argument. This argument goes back to the 1970 thesis of Margulis [23] and was generalized by Eskin and McMullen [13]. For $n=2$, Sarnak [34] obtained a sharper result, based on the study of Eisenstein series.

(2) In principle, our methods should extend to prove an analogous result for $G=\operatorname{Isom}^{+}\left(\mathbb{H}^{n}\right)$ for any $n \geq 2$; however computations needed to understand $\phi_{\ell}$ 's seem very intricate as the dimension gets higher.

(3) When $\psi$ is $K$-invariant, Theorem 1.3 was obtained in [20]. See also [18] for its extensions to other rank one Lie groups.

As mentioned before, our approach in proving Theorem 1.3 is based on the existence of $L^{2}$-eigenfunctions on $\Gamma \backslash \mathbb{H}^{n}$ for $\delta>(n-1) / 2$ and hence cannot be applied to $\Gamma$ with $\delta \leq(n-1) / 2$. However a noneffective version of Theorem 1.3 is available for any $\delta>0$; this was obtained in [30] when $(N \cap \Gamma) \backslash N$ is compact and in [26] in general. In these papers, the coefficient of the main term was given in terms of the Burger-Roblin measure associated to the stable horospherical foliation. In applications to counting problems, it is much handier to have this coefficient in terms of a measure instead of an infinite sum. For this reason, we present an alternative formulation of Theorem 1.3 in Theorem 1.6.

1.4. Equidistribution in ergodic terms. Let $\nu_{j}$ denote the PattersonSullivan measure on the limit set $\Lambda(\Gamma)$ associated to the basepoint $j=\left(0_{n-1}, 1\right) \in \mathbb{H}^{n}$, which is unique up to a constant multiple.

Sullivan gave an explicit construction of the base eigenfunction $\phi_{0} \in$ $L^{2}(\Gamma \backslash G)^{K}$ using $\nu_{j}$ :

$$
\phi_{0}\left(n_{x} a_{y}\right)=\int_{u \in \mathbb{R}^{n-1}}\left(\frac{\left(|u|^{2}+1\right) y}{|x-u|^{2}+y^{2}}\right)^{\delta} d \nu_{j}(u) .
$$

Here and also later, we identify $\mathbb{C}=\mathbb{R}^{2}$ for $n=3$, so that $|x-u|^{2}=$ $\left(x_{1}-u_{1}\right)^{2}+\left(x_{2}-u_{2}\right)^{2}$ for $x=x_{1}+i x_{2}$ and $u=\left(u_{1}, u_{2}\right)$. We normalize $\nu_{j}$ so that $\left\|\phi_{0}\right\|_{2}=1$ [37]. 
Define the measure $\tilde{m}_{N}^{\mathrm{BR}}$ on $G$ in the Iwasawa coordinates $G=K A N$ : for $\psi \in C_{c}(G)$,

$$
\tilde{m}_{N}^{\mathrm{BR}}(\psi)=\int_{K A N} \psi\left(k a_{y} n_{x}\right) y^{\delta-1} d x d y d \nu_{j}(k(0)) .
$$

This measure is left $\Gamma$-invariant and right $N$-invariant, and the BurgerRoblin measure $m_{N}^{\mathrm{BR}}$ (associated to the stable horospherical subgroup $N)$ is the measure on $\Gamma \backslash G$ induced from $\tilde{m}_{N}^{\mathrm{BR}}$. The BR measure $m_{N}^{\mathrm{BR}}$ is an infinite measure whenever $0<\delta<n-1$ [26].

Theorem 1.6. Let $n=2$ or 3 and $(n-1) / 2<\delta \leq n-1$. For any $\psi \in C_{c}^{\infty}(\Gamma \backslash G)^{M}$, as $y \rightarrow 0$,

$$
\begin{aligned}
\int_{(N \cap \Gamma) \backslash N} \psi\left(n_{x} a_{y}\right) d x=\kappa_{\Gamma} \cdot m_{N}^{\mathrm{BR}}(\psi) & \cdot y^{n-1-\delta} \\
& +O\left(\mathcal{S}_{2 n-1}(\psi) \cdot y^{(n-1-\delta)+\frac{2 \mathbf{s}_{\Gamma}}{2 n+1}}\right)
\end{aligned}
$$

where $\kappa_{\Gamma}=\int_{x \in \mathbb{R}^{n-1}}\left(1+|x|^{2}\right)^{-\delta} d x \cdot \int_{n_{x} \in(N \cap \Gamma) \backslash N}\left(1+|x|^{2}\right)^{\delta} d \nu_{j}(x)$.

1.5. Effective orbital counting and Affine sieves in sectors. Let $Q$ be a quadratic form over $\mathbb{Q}$ of signature $(n, 1)$ and $v_{0} \in \mathbb{Z}^{n+1}$ a nonzero vector such that $Q\left(v_{0}\right)=0$. Let $G_{0}$ denote the identity component of $\mathrm{SO}_{Q}(\mathbb{R})$. As well known, $G_{0}$ is isomorphic to $G=\mathrm{PSL}_{2}(\mathbb{R}$ ) (for $n=2$ ) and $G=\mathrm{PSL}_{2}(\mathbb{C})$ (for $n=3$ ) as real Lie groups. Let $\Gamma<G_{0}(\mathbb{Z})$ be a geometrically finite subgroup with $\delta>(n-1) / 2$ such that $v_{0} \Gamma$ is discrete. For each square-free integer $d$, let $\Gamma_{d}$ be a subgroup of $\Gamma$ containing $\{\gamma \in \Gamma: \gamma \equiv e(\bmod \mathrm{d})\}$ and satisfying $\operatorname{Stab}_{\Gamma} v_{0}=\operatorname{Stab}_{\Gamma_{d}} v_{0}$.

By a theorem of Bourgain, Gamburd and Sarnak [5], there exists a uniform spectral gap, say $\mathbf{s}_{0}>0$, for all $\Gamma_{d}, d$ square-free.

Consider the representation $G \rightarrow G_{0}$ such that $N$ is contained in $\operatorname{Stab}_{G}\left(v_{0}\right)$, and fix a norm $\|\cdot\|$ on $\mathbb{R}^{n+1}$. For any subset $\Omega \subset K$ and $T>0$, define the sector

$$
S_{T}(\Omega):=\left\{v \in v_{0} A \Omega:\|v\|<T\right\} .
$$

Define $q_{\Omega}$ to be the maximum of $0 \leq q \leq 1$ such that

$$
\nu_{j}\left(\epsilon \text {-neighborhood of } \partial\left(\Omega^{-1}(0)\right)\right) \ll \epsilon^{q} \text { for all small } \epsilon>0 \text {. }
$$

Note that if $\partial\left(\Omega^{-1}(0)\right) \cap \Lambda(\Gamma)=\emptyset$, then $q_{\Omega}=1$. We will say $\Omega$ admissible if $q_{\Omega}>0$. 
Theorem 1.8. Let $\Omega$ be an admissible left $M$-invariant Borel subset of $K$. Then for any $\gamma \in \Gamma$, as $T \rightarrow \infty$,

$$
\#\left\{v \in v_{0} \Gamma_{d} \gamma \cap S_{T}(\Omega)\right\}=\frac{\Xi_{v_{0}}(\Gamma, \Omega)}{\left[\Gamma: \Gamma_{d}\right]} \cdot T^{\delta}+O\left(T^{\delta-\frac{8 \mathbf{s}_{0}}{n(n+9)(2 n+1) q_{\Omega}}}\right) .
$$

Identifying $\Gamma$ with its pull back in $G, \Xi_{v_{0}}(\Gamma, \Omega)$ is given by

$$
\Xi_{v_{0}}(\Gamma, \Omega)=\kappa_{\Gamma} \int_{k \in \Omega^{-1}} \frac{d \nu_{j}(k(0))}{\left\|v_{0} k^{-1}\right\|^{\delta}} .
$$

As $\nu_{j}$ is supported on the limit set $\Lambda(\Gamma), \Xi_{v_{0}}(\Gamma, \Omega)>0$ if and only if the interior of $\Omega^{-1}(0)$ intersects $\Lambda(\Gamma)$.

Given an integer-valued polynomial $F$ on the orbit $v_{0} \Gamma$, Theorem 1.8 has an application in studying integral points $\mathbf{x}$ lying in a fixed sector with $F(\mathbf{x})$ having at most $R$ prime factors (including multiplicities). For instance, the following theorem can be deduced from Theorem 1.8 using the same analysis as in [20, section 8].

Theorem 1.10. Suppose that $\Omega \subset K$ be an admissible subset such that the interior of $\Omega^{-1}(0)$ intersects $\Lambda(\Gamma)$. Then there exists $R \geq 1$ (depending on $\mathbf{s}_{\mathbf{0}}$ ) such that for each $1 \leq i \leq n+1$,

$$
\#\left\{\mathbf{x} \in v_{0} \Gamma \cap S_{T}(\Omega): x_{1} \cdots x_{i} \text { has at most } R \text { prime factors }\right\} \asymp \frac{T^{\delta}}{(\log T)^{i}}
$$

where $\mathbf{x}=\left(x_{1}, \cdots, x_{n+1}\right)$ and $f(T) \asymp g(T)$ means that their ratio is between two positive constants uniformly for all $T \gg 1$.

Theorem 1.8 is proved in 26] without an error term. When the norm is $K$-invariant and $\Omega=K$, it was also proved in [20]. Theorem 1.10 for $\Omega=K$ has been obtained in [20] (also see [21]).

1.6. Organization: Sections 2-4 are devoted to understanding the base eigenfunctions $\phi_{\ell}$ 's and their integrals over closed $N$-orbits. In section 2, we find a computable recursive formula (Theorem 2.11) for a raising operator among $M$-invariant vectors in a general complementary series representation of $G$. Using this, in section 3, we obtain an explicit description of $\phi_{\ell}$ 's which turn out to be related to the Legendre polynomials for $n=3$. Understanding each $\phi_{\ell}$ as a function of $\Gamma \backslash G$, rather than as a vector in the Hilbert space $L^{2}(\Gamma \backslash G)$, is crucial in our approach, as we need to deal with several convergence issues of the integrals of $\phi_{\ell}$ 's as well as to thicken the $N$-integrals of $\phi_{\ell}$ 's uniformly over all $\ell$ 's. In section 4 , we compute the $N$-integrals of $\phi_{\ell}$ 's and compute $c_{n}(\ell)$ 's explicitly (modulo $c_{0}$ ). In section 5 , we carry out the thickening of the $N$-integrals of $\phi_{\ell}$ 's uniformly. Since $\phi_{\ell}$ 's are not supported on compact subsets of $\Gamma \backslash G$, this step is delicate, as we need to ensure 
that there is at most a polynomial error term in $\ell$ in this procedure. The equidistribution theorems 1.3 and 1.6 are proved in section 6 and 7 respectively. In section 8 , we deduce Theorem 1.8 and Theorem 1.1 from Theorem 1.6.

Added in print: Soon after we submitted the first version of our paper to the arXive, we received a preprint by Vinogradov [38], which also proves Theorem 1.1 (with a weaker error term) using different methods.

Acknowledgment: We thank Peter Sarnak for useful comments on the preliminary version of this paper.

\section{LADDER OPERATORS}

2.1. Notations and Preliminaries. Let $G$ be $\mathrm{PSL}_{2}(\mathbb{R})$ or $\mathrm{PSL}_{2}(\mathbb{C})$. Hence as a real Lie group, $G$ is isomorphic to the identity component of $\mathrm{SO}(n, 1)$ for $n=2$ and 3 respectively. In this subsection, we introduce notations which will be used throughout the paper and review some basic facts about representations of $G$. Let $K$ be a maximal compact subgroup of $G$. Denoting by $\mathfrak{g}$ and $\mathfrak{k}$ the Lie algebras of $G$ and $K$ respectively, let $\mathfrak{g}=\mathfrak{k} \oplus \mathfrak{p}$ be the corresponding Cartan decomposition of $\mathfrak{g}$. Let $A=\exp (\mathfrak{a})$ where $\mathfrak{a}$ is a maximal abelian subspace of $\mathfrak{p}$ and let $M$ be the centralizer of $A$ in $K$.

Define the symmetric bi-linear form $\langle\cdot, \cdot\rangle$ on $\mathfrak{g}$ by

$$
\langle X, Y\rangle:=\frac{1}{2(n-1)} B(X, Y)
$$

where $B(X, Y)=\operatorname{Tr}(\operatorname{ad} X \operatorname{ad} Y)$ denotes the Killing form for $\mathfrak{g}$. The reason for this normalization is so that the Riemmanian metric on $G / K$ induced by $\langle\cdot, \cdot\rangle$ has constant curvature -1 .

Let $\left\{X_{i}\right\}$ be a basis for $\mathfrak{g}_{\mathbb{C}}$ over $\mathbb{C}$; put $g_{i j}=\left\langle X_{i}, X_{j}\right\rangle$ and let $g^{i j}$ be the $(i, j)$ entry of the inverse matrix of $\left(g_{i j}\right)$. The element

$$
\mathcal{C}=\sum g^{i j} X_{i} X_{j}
$$

is called the Casimir element of $\mathfrak{g}_{\mathbb{C}}$ (with respect to $\langle\cdot, \cdot\rangle$ ). It is wellknown that this definition is independent of the choice of a basis and that $\mathcal{C}$ lies in the center of the universal enveloping algebra $U\left(\mathfrak{g}_{\mathbb{C}}\right)$ of $\mathfrak{g}_{\mathbb{C}}$.

For $G=\mathrm{PSL}_{2}(\mathbb{R})$, set

$$
K=\operatorname{PSO}(2)=\left\{k_{\theta}=\left(\begin{array}{cc}
\cos \theta & \sin \theta \\
-\sin \theta & \cos \theta
\end{array}\right): \theta \in[0, \pi)\right\} ;
$$


and for $G=\mathrm{PSL}_{2}(\mathbb{C})$, we set $K=\mathrm{PSU}(2)$. In both cases, we set $N$ to be the strict upper triangular subgroup of $G$ and $A$ the diagonal subgroup consisting of positive diagonals. We have the Iwasawa decomposition $G=N A K$ : any element $g$ of $G$ is written uniquely as $g=n_{x} a_{y} k$ where $n_{x}=\left(\begin{array}{cc}1 & x \\ 0 & 1\end{array}\right) \in N, a_{y}=\left(\begin{array}{cc}\sqrt{y} & 0 \\ 0 & \sqrt{y}^{-1}\end{array}\right)$ and $k \in K$. Note that $M=\{e\}$ and $M=\left\{\left(\begin{array}{cc}e^{i \theta} & 0 \\ 0 & e^{-i \theta}\end{array}\right): \theta \in[0, \pi)\right\}$ respectively.

Set $\mathbb{H}^{n}=\left\{(x, y): x \in \mathbb{R}^{n-1}, y>0\right\}$ and $j=\left(0_{n-1}, 1\right)$. The group $G$ acts on $\mathbb{H}^{n}$ via the extension of the Möbius transformation action on the boundary $\mathbb{R}^{n-1} \cup\{\infty\}$. Under this action, we have $K=\operatorname{Stab}_{G}(j)$ and $\mathbb{H}^{n}=G / K \simeq \exp (\mathfrak{p})$. Now the Laplacian operator $\Delta$ on $\mathbb{H}^{n}$ is respectively given by

$$
\Delta=-y^{2}\left(\frac{\partial^{2}}{\partial x^{2}}+\frac{\partial^{2}}{\partial y^{2}}\right) \text { and } \Delta=-y^{2}\left(\frac{\partial^{2}}{\partial x_{1}^{2}}+\frac{\partial^{2}}{\partial x_{2}^{2}}+\frac{\partial^{2}}{\partial y^{2}}\right)+y \frac{\partial}{\partial y}
$$

according as $n=2$ or 3. By Kuga's lemma, for all $\psi \in C^{\infty}(G)^{K}=$ $C^{\infty}\left(\mathbb{H}^{n}\right)$, we have

$$
\mathcal{C}(\psi)=-\Delta(\psi)
$$

Consider the following elements of $\mathfrak{g}$ :

$$
H=\left(\begin{array}{cc}
1 & 0 \\
0 & -1
\end{array}\right), \quad E=\left(\begin{array}{ll}
0 & 1 \\
0 & 0
\end{array}\right) \text { and } F=\left(\begin{array}{ll}
0 & 0 \\
1 & 0
\end{array}\right) .
$$

For $G=\operatorname{PSL}_{2}(\mathbb{R})$, since $\{H, E, F\}$ is a basis for $\mathfrak{g}_{\mathbb{C}}$, we can compute $\mathcal{C}$ by a direct method and obtain:

Lemma 2.1. For $n=2$, we have

$$
\mathcal{C}=\frac{1}{4} H^{2}+\frac{1}{2}(E F+F E) .
$$

We set $\mathcal{C}_{K}=E-F=\frac{\partial}{\partial \theta}$.

To compute the Casimir element for $n=3$, note that the Lie algebra of $K=\operatorname{PSU}(2)$ is

$$
\mathfrak{k}=\left\{X \in \mathrm{M}_{2}(\mathbb{C}): \bar{X}^{t}=-X, \operatorname{tr}(X)=0\right\} .
$$

The elements $\frac{i}{2} H, X_{1}:=\frac{1}{2}(E-F), X_{2}:=\frac{i}{2}(E+F)$ generate $\mathfrak{k}$ as a real vector space. Set

$$
D:=\frac{i}{2} H \in \mathfrak{k}_{\mathbb{C}}, \quad E^{+}:=X_{1}-i X_{2} \in \mathfrak{k}_{\mathbb{C}} \text { and } E^{-}:=-X_{1}-i X_{2} \in \mathfrak{k}_{\mathbb{C}} .
$$

The elements $\frac{1}{2} H, Y_{1}:=\frac{1}{2}(E+F)$ and $Y_{2}:=\frac{i}{2}(E-F)$ form a basis of $\mathfrak{p}$ over $\mathbb{R}$. Set

$$
\tilde{H}:=\frac{1}{2} H \in \mathfrak{p}_{\mathbb{C}}, \quad R:=Y_{1}-i Y_{2} \in \mathfrak{p}_{\mathbb{C}} \text { and } L:=-Y_{1}-i Y_{2} \in \mathfrak{p}_{\mathbb{C}}
$$


Lemma 2.4. For $n=3$, we have

$$
\mathcal{C}=\mathcal{C}_{K}+\tilde{H}^{2}-\frac{1}{2}(R L+L R)
$$

where $\mathcal{C}_{K}:=-D^{2}+\frac{1}{2}\left(E^{+} E^{-}+E^{-} E^{+}\right)$is the Casimir element of $\mathfrak{k}_{\mathbb{C}}$ (up to a constant multiple).

Proof. Note that $\left\{D, E^{+}, E^{-}, \tilde{H}, R, L\right\}$ forms a basis of $\mathfrak{g}_{\mathbb{C}}$. We check $\left[D, E^{ \pm}\right]= \pm i E^{ \pm},[D, R]-i R,[D, L]=-i L,\left[E^{+}, E^{-}\right]=-2 i D$, $\left[E^{+}, R\right]=\left[E^{-}, L\right]=[D, \tilde{H}]=0,\left[E^{-}, \tilde{H}\right]=-L,\left[E^{+}, \tilde{H}\right]=-R$, $\left[E^{-}, R\right]=-2 \tilde{H},\left[E^{+}, L\right]=-2 \tilde{H},[R, \tilde{H}]=-E^{+},[L, \tilde{H}]=E^{-}$, and $[R, L]=2 i D$.

Using these relations, we can compute the matrix $g_{i j}$ used in the definition of $\mathcal{C}$ and obtain the above formula for $\mathcal{C}$. Since $\left\{D, E^{+}, E^{-}\right\}$ forms a basis of $\mathfrak{k}_{\mathbb{C}}$, we compute that $-D^{2}+\frac{1}{2}\left(E^{+} E^{-}+E^{-} E^{+}\right)$is the Casimir element of $\mathfrak{k}_{\mathbb{C}}$ (up to a scalar multiple) using the above relations.

2.2. Complementary series Representation of $G$. Let $V$ be an infinite dimensional irreducible unitary representation of $G$. Denote by $V^{K}$ and $V^{M}$ the subspaces of $K$-invariant and $M$-invariant vectors respectively. We assume that $V^{K}$ is non-trivial and fix a unit vector in $v_{0} \in V^{K}$, which is unique up to a scalar multiple.

Let $V^{\infty}$ denote the set of smooth vectors of $V$, i.e., $v \in V^{\infty}$ if the map $g \mapsto g v$ is a smooth function $G \rightarrow V$. Every element of $\mathfrak{g}$ acts as a differential operator on $V^{\infty}$ : for $X \in \mathfrak{g}$ and $v \in V^{\infty}$,

$$
\pi(X)(v):=\left.\frac{d}{d t}(\exp (t X) \cdot v)\right|_{t=0}
$$

where $\exp (X)=\sum_{j=0}^{\infty} \frac{X^{j}}{j !}$ denotes the usual exponential map $\mathfrak{g} \rightarrow G$. This action extends to the action of the universal enveloping algebra $U\left(\mathfrak{g}_{\mathbb{C}}\right)$ on $V^{\infty}$.

Denote by $\hat{G}$ the unitary dual of $G$. A representation $\pi \in \hat{G}$ is called tempered if for any $K$-finite vectors $v, w$ of $\pi$, the matrix coefficient function $g \mapsto\langle\pi(g) v, w\rangle$ belongs to $L^{2+\epsilon}(G)$ for any $\epsilon>0$. It follows from the classification of $\hat{G}$ (cf. [19, Thm. 16.2-3]) that the nontempered spectrum of $\hat{G}$ consists of the trivial representation and the complementary series representation $\left(\pi_{s}, \mathcal{H}_{s}\right)$ parametrized by $\frac{n-1}{2}<$ $s<(n-1)$, where $\mathcal{C}$ acts on $\mathcal{H}_{s}^{\infty}$ by the scalar $s(s-n+1)$.

We fix $V=\mathcal{H}_{s}$ for $\frac{n-1}{2}<s<(n-1)$. If $n=2$, the unitary dual $\hat{K}$ can be parametrized by $\mathbb{Z}$ so that $\ell \in \hat{K}$ corresponds to the one dimensional representation $V_{\ell}$ on which $\mathcal{C}_{K}$ acts by the scalar $-4 \ell^{2}$. 
For $n=3, \hat{K}$ can be parametrized by $\mathbb{Z}_{\geq 0}$ so that $\ell \in \hat{K}$ corresponds to the irreducible $2 \ell+1$ dimensional representation $V_{\ell}$.

Lemma 2.5. For each $\ell \geq 0, \mathcal{C}_{K}$ acts on $V_{\ell}$ as the scalar $\ell(\ell+1)$.

Proof. If $w_{\ell}$ is the highest weight vector of $V_{\ell}$, then $D\left(w_{\ell}\right)=i \ell w_{\ell}$. Using $\left[E^{+}, E^{-}\right]=-2 i D$, we can write $\mathcal{C}_{K}=-D^{2}-i D+E^{-} E^{+}$. Hence $\mathcal{C}_{K}\left(w_{\ell}\right)=\ell^{2} w_{\ell}+\ell w_{\ell}$ since $E^{+}\left(w_{\ell}\right)=0$. Since $\mathcal{C}_{K}$ acts by the scalar on $V_{\ell}$ as $V_{\ell}$ is irreducible, the claim follows.

As a $K$-representation, we write

$$
V=\oplus_{\ell \in \hat{K}} m_{\ell} V_{\ell}
$$

where the multiplicity $m_{\ell}$ of $V_{\ell}$ is at most one for each $\ell$ (see the remark following Theorem 4.5 of [39]); in fact we show in the next subsection that $m_{\ell}=1$ for all $\ell \in \hat{K}$. We also have that the space $V_{\ell}^{M}$ is at most one dimensional [11].

2.3. Ladder operators. In this subsection, we will compute the Ladder operators which maps $V_{\ell}^{M}$ to $V_{\ell+1}^{M}$ and use them to obtain an explicit recursive formula for a unit vector of $V_{\ell}^{M}$ starting from $v_{0}$. These are well-known for $n=2$.

\subsubsection{The case $G=\mathrm{PSL}_{2}(\mathbb{R})$. Set}

$$
\mathcal{R}=\frac{1}{2}(\pi(H)+i \pi(E+F)) \quad \text { and } \quad \overline{\mathcal{R}}=\frac{1}{2}(\pi(H)-i \pi(E+F)) .
$$

These are called raising and lowering operators respectively and it is well-known that $\mathcal{R}\left(V_{\ell}\right)=V_{\ell+1}$ and $\overline{\mathcal{R}}\left(V_{\ell}\right)=V_{\ell-1}$ for any $\ell \in \mathbb{Z}$ (cf. [1, Prop. 2.5.2]. For a fixed unit vector $v_{0} \in V^{K}$, put

$$
v_{\ell}= \begin{cases}\mathcal{R}^{\ell}\left(v_{0}\right) & \text { if } \ell \geq 0 \\ \overline{\mathcal{R}}^{|\ell|}\left(v_{0}\right) & \text { if } \ell<0 .\end{cases}
$$

Then $V=\oplus_{\ell \in \hat{K}} \mathbb{C} v_{\ell}$ and for $\ell \geq 0$, we have (see [7]):

$$
\left\|v_{ \pm \ell}\right\|_{2}=\frac{\sqrt{\Gamma(s+\ell) \Gamma(1-s+\ell)}}{\sqrt{\Gamma(s) \Gamma(1-s)}} .
$$

where $\Gamma(x)$ denotes the Gamma function and $\|v\|$ denotes the norm of $v \in V:\|v\|=\sqrt{\langle v, v\rangle}$. 
2.3.2. The case $G=\mathrm{PSL}_{2}(\mathbb{C})$. Recall the elements $D, E^{+}, E^{-}, \tilde{H}, R, L$ from $(2.2)$ and $(2.3)$. For each $\ell \geq 0$, the $K$-space $V_{\ell}$ is the irreducible representation of $K$ of dimension $2 \ell+1$. The operators $E^{ \pm}$move between different $M$-types inside each fixed $V_{\ell}$ and $R$ (resp. $L$ ) maps the highest (resp. lowest) weight vector space of each $V_{\ell}$ into the highest (resp. lowest) weight vector space of $V_{\ell+1}$.

We will show that the following differential operator $\mathcal{Z}_{\ell}$ maps $V_{\ell}^{M}$ to $V_{\ell+1}^{M}$ : for each $\ell \geq 0$, set

$$
\mathcal{Z}_{\ell}:=\frac{1}{2}\left(R E^{-}+L E^{+}-2(\ell+1) \tilde{H}\right) .
$$

Lemma 2.7. For each $\ell \in \mathbb{Z}_{\geq 0}$,

(1) $\mathcal{C}_{K} \mathcal{Z}_{\ell}=\mathcal{Z}_{\ell} \mathcal{C}_{K}-i\left(R E^{-}-L E^{+}\right) D-2 \tilde{H}\left(\mathcal{C}_{K}+D^{2}\right)+2(\ell+1)\left(\mathcal{Z}_{\ell}+\right.$ $\ell \tilde{H})$

(2) $D \mathcal{Z}_{\ell}=\mathcal{Z}_{\ell} D$

(3) $\mathcal{Z}_{\ell} \tilde{H}=\tilde{H} \mathcal{Z}_{\ell}+\mathcal{C}-2 \mathcal{C}_{K}-D^{2}-\tilde{H}^{2}$.

Proof. Since $\mathcal{C}_{K}=-D^{2}+\frac{1}{2}\left(E^{+} E^{-}+E^{-} E^{+}\right)$, we compute

(1) $\mathcal{C}_{K} \tilde{H}=\tilde{H} \mathcal{C}_{K}-2 \mathcal{Z}_{\ell}-2 \ell \tilde{H}$

(2) $\mathcal{C}_{K} R=R \mathcal{C}_{K}-2 i D R-2 \tilde{H} E^{+}$, and

(3) $\mathcal{C}_{K} L=L \mathcal{C}_{K}+2 i D L-2 \tilde{H} E^{-}$.

These relations imply

$$
\begin{aligned}
& \mathcal{C}_{K} \mathcal{Z}_{\ell}=\frac{1}{2}\left(R E^{-}+L E^{+}-2(\ell+1) \tilde{H}\right) \mathcal{C}_{K} \\
& -\left\{i D R E^{-}-i D L E^{+}+\tilde{H}\left(E^{+} E^{-}+E^{-} E^{+}\right)-2(\ell+1)\left(\mathcal{Z}_{\ell}+\ell \tilde{H}\right)\right\} .
\end{aligned}
$$

Using $E^{+} E^{-}=\mathcal{C}_{K}+D^{2}-i D, E^{-} E^{+}=\mathcal{C}_{K}+D^{2}+i D, D R E^{-}=R E^{-} D$ and $D L E^{+}=L E^{+} D$, we compute that $\mathcal{C}_{K} \mathcal{Z}_{\ell}$ is equal to

$\mathcal{Z}_{\ell} \mathcal{C}_{K}-i\left(R E^{-} D-L E^{+} D\right)-\tilde{H}\left(2 \mathcal{C}_{K}+2 D^{2}\right)+2(\ell+1) \mathcal{Z}_{\ell}+2(\ell+1) \ell \tilde{H}$.

For $(2)$, we note that $[D, R]=i R,[D, L]=-i L,[\tilde{H}, D]=0$, $\left[D, E^{ \pm}\right]= \pm i E^{ \pm}$. Hence

$$
\begin{aligned}
D \mathcal{Z}_{\ell} & =\frac{1}{2}\left(D R E^{-}+D L E^{+}-2(\ell+1) D \tilde{H}\right) \\
& =\frac{1}{2}\left\{(i R+R D) E^{-}+(-i L+L D) E^{+}-2(\ell+1) \tilde{H} D\right\} \\
& =\frac{1}{2}\left\{R E^{-}+L E^{+}-2(\ell+1) \tilde{H}\right\} D=\mathcal{Z}_{\ell} D .
\end{aligned}
$$

(3) can be proved similarly using $\mathcal{C}=\mathcal{C}_{K}+\frac{1}{2}\left(2 \tilde{H}^{2}-R L-L R\right)$.

Proposition 2.8. For each $\ell \geq 0$,

$$
\mathcal{Z}_{\ell}\left(V_{\ell}^{M}\right) \subset V_{\ell+1}^{M}
$$


Proof. Note that $v \in V_{\ell}$ if and only if $\mathcal{C}_{K} v=\ell(\ell+1) v$, and $v \in V^{M}$ if and only if $D v=0$.

Let $v \in V_{\ell}^{M}$. Using $D v=0$ and Lemma 2.7 (1), we deduce that

$$
\begin{aligned}
\mathcal{C}_{K}\left(\mathcal{Z}_{\ell} v\right) & =\ell(\ell+1) \mathcal{Z}_{\ell} v-2 \ell(\ell+1) \tilde{H} v+2(\ell+1) \mathcal{Z}_{\ell} v+2 \ell(\ell+1) \tilde{H} v \\
& =(\ell+1)(\ell+2) \mathcal{Z}_{\ell} v
\end{aligned}
$$

Hence $\mathcal{Z}_{\ell} v \in V_{\ell+1}$. By Lemma 2.7 (2), we have

$$
D \mathcal{Z}_{\ell} v=\mathcal{Z}_{\ell}(D v)=0
$$

and hence $\mathcal{Z}_{\ell} v \in V_{\ell+1}^{M}$.

Fixing a unit vector $v_{0} \in V^{K}$, define $v_{\ell}, \ell \geq 1$, recursively:

$$
v_{\ell}:=\mathcal{Z}_{\ell}\left(v_{\ell-1}\right) \text {. }
$$

Put

$$
a_{\ell}:=-2 \ell+1 \quad \text { and } \quad b_{\ell}:=(\ell-1)^{2}(\ell(\ell-2)-s(s-2)) .
$$

Theorem 2.11 (Recursive formula for $v_{\ell}$ ). For $\ell \geq 1$,

$$
v_{\ell}=a_{\ell} \tilde{H} v_{\ell-1}+b_{\ell} v_{\ell-2}
$$

where $v_{-1}$ is understood as the zero vector.

Proof. For $\ell \geq 0$ and $m \geq 0$, we have

$$
\begin{aligned}
\mathcal{Z}_{\ell} & =\frac{1}{2}\left(R E^{-}+L E^{+}-2(\ell+1) \tilde{H}\right) \\
& =\frac{1}{2}\left(R E^{-}+L E^{+}-2(m+1) \tilde{H}+(-2(\ell+1)+2(m+1)) \tilde{H}\right) \\
& =\mathcal{Z}_{m}+(m-\ell) \tilde{H}
\end{aligned}
$$

and hence

$$
\mathcal{Z}_{\ell} v_{m}=v_{m+1}+(m-\ell) \tilde{H} v_{m} .
$$

We need to show that for $\ell \geq 1$,

$$
v_{\ell}=a_{\ell} \tilde{H} v_{\ell-1}+b_{\ell} v_{\ell-2} \text {. }
$$

Since $E^{+} v_{0}=E^{-} v_{0}=0$, we have $v_{1}=\mathcal{Z}_{0} v_{0}=-\tilde{H} v_{0}$. For the induction process, assume $v_{\ell}=a_{\ell} \tilde{H} v_{\ell-1}+b_{\ell} v_{\ell-2}$. We deduce that, using Lemma 2.7 (3) and 2.12,

$$
\begin{aligned}
v_{\ell+1} & =\mathcal{Z}_{\ell} v_{\ell} \\
& =a_{\ell}\left(\mathcal{Z}_{\ell} \tilde{H} v_{\ell-1}\right)+b_{\ell}\left(\mathcal{Z}_{\ell} v_{\ell-2}\right) \\
& =a_{\ell}\left(\tilde{H} \mathcal{Z}_{\ell}+\mathcal{C}-2 \mathcal{C}_{K}-D^{2}-\tilde{H}^{2}\right) v_{\ell-1}+b_{\ell}\left(v_{\ell-1}-2 \tilde{H} v_{\ell-2}\right) .
\end{aligned}
$$

Observe that $\mathcal{C} v_{\ell-1}=\lambda v_{\ell-1}$ with $\lambda=-s(2-s), \mathcal{C}_{K} v_{\ell-1}=\ell(\ell-1) v_{\ell-1}$ and $D v_{\ell-1}=0$. 
Using the induction hypothesis, we deduce

$$
\begin{aligned}
v_{\ell+1} & =a_{\ell}\left(\tilde{H}\left(v_{\ell}-\tilde{H} v_{\ell-1}\right)+(\lambda-2 \ell(\ell-1)) v_{\ell-1}-\tilde{H}^{2} v_{\ell-1}\right) \\
& +b_{\ell}\left(v_{\ell-1}-2 \tilde{H} v_{\ell-2}\right) \\
& =a_{\ell} \tilde{H} v_{\ell}-2 \tilde{H}\left(a_{\ell} \tilde{H} v_{\ell-1}+b_{\ell} v_{\ell-2}\right)+\left(a_{\ell}(\lambda-2 \ell(\ell-1))+b_{\ell}\right) v_{\ell-1} \\
& =\left(a_{\ell}-2\right) \tilde{H} v_{\ell}+\left(a_{\ell}(\lambda-2 \ell(\ell-1))+b_{\ell}\right) v_{\ell-1} \\
& =a_{\ell+1} \tilde{H} v_{\ell}+b_{\ell+1} v_{\ell-1} .
\end{aligned}
$$

This finishes the proof.

For $x>0$, the Gamma function $\Gamma(x)$ is defined to be the integral $\int_{0}^{\infty} e^{-t} t^{-x-1} d t$ : it satisfies $\Gamma(x+1)=x \Gamma(x)$ and for each positive integer $\ell, \Gamma(\ell)=\ell !$.

Lemma 2.13. For each $\ell \geq 0$,

$$
\left\|v_{\ell}\right\|=\frac{\ell !}{\sqrt{2 \ell+1}} \frac{\sqrt{\Gamma(s+\ell) \Gamma(2-s+\ell)}}{\sqrt{\Gamma(s) \Gamma(2-s)}} .
$$

In particular, $v_{\ell} \neq 0$ for each $\ell \geq 0$.

Proof. Note that $v_{\ell}$ 's are mutually orthogonal to each other. By Lemma 2.11, we have $v_{\ell}=a_{\ell} \tilde{H} v_{\ell-1}+b_{\ell} v_{\ell-2}$. Therefore

$$
\begin{aligned}
\left\|v_{\ell}\right\|^{2} & =\left\langle a_{\ell} \tilde{H} v_{\ell-1}+b_{\ell} v_{\ell-2}, v_{\ell}\right\rangle=\left\langle a_{\ell} \tilde{H} v_{\ell-1}, v_{\ell}\right\rangle \\
& =-\left\langle a_{\ell} v_{\ell-1}, \tilde{H} v_{\ell}\right\rangle=-\left\langle a_{\ell} v_{\ell-1}, \frac{1}{a_{\ell+1}} v_{\ell+1}-\frac{b_{\ell+1}}{a_{\ell+1}} v_{\ell-1}\right\rangle \\
& =\frac{a_{\ell} b_{\ell+1}}{a_{\ell+1}}\left\|v_{\ell-1}\right\|^{2} .
\end{aligned}
$$

It follows that

$$
\begin{aligned}
\left\|v_{\ell}\right\|^{2} & =\frac{a_{\ell} b_{\ell+1}}{a_{\ell+1}} \cdot \frac{a_{\ell-1} b_{\ell}}{a_{\ell}} \cdots \frac{a_{\ell+1-j} b_{\ell+2-j}}{a_{\ell+2-j}} \cdots \frac{a_{1} b_{2}}{a_{2}}\left\|v_{0}\right\|^{2} \\
& =\frac{a_{1}}{a_{\ell+1}} \cdot \prod_{j=1}^{\ell} b_{j+1} \\
& =\frac{1}{2 \ell+1} \prod_{j=1}^{\ell} j^{2}\left(j^{2}-1-s(s-2)\right) \\
& =\frac{(\ell !)^{2}}{2 \ell+1} \prod_{j=1}^{\ell}(j+s-1)(j-s+1) .
\end{aligned}
$$


Therefore the claim follows by the well-known properties of the Gamma function.

Lemma 2.13 shows that $\mathcal{Z}_{\ell}$ maps a non-zero vector to a non-zero vector. Therefore Theorem 2.11 together with Lemma 2.13 implies the following:

Corollary 2.14. For each $\ell \in \mathbb{Z}_{\geq 0}$,

$$
\mathcal{Z}_{\ell}\left(V_{\ell}^{M}\right)=V_{\ell+1}^{M} \quad \text { and } \quad V^{M}=\oplus_{\ell \in \hat{K}} \mathbb{C} v_{\ell} .
$$

\section{EXPLICIT FORMULAS FOR BASE EIGENFUNCTIONS $\phi_{\ell}$}

Let $G=\mathrm{PSL}_{2}(\mathbb{R})$ or $\mathrm{PSL}_{2}(\mathbb{C})$, so that $G=\mathrm{Isom}^{+}\left(\mathbb{H}^{n}\right)$ for $n=2,3$ respectively. We keep the notations for $N, A, M, \mathcal{C}$ and $\Delta$, etc. from the section 2. In particular, $\mathcal{C}$ satisfies $\mathcal{C}(\psi)=-\Delta(\psi)$ for all $\psi \in$ $C^{\infty}(\Gamma \backslash G)^{K}=C^{\infty}\left(\mathbb{H}^{n}\right)$.

Let $\Gamma<G$ be a geometrically finite discrete subgroup with critical exponent $\frac{n-1}{2}<\delta<n-1$. Let $\nu_{j}=\nu_{j}(\Gamma)$ denote the PattersonSullivan measure on the limit set $\Lambda(\Gamma)$ with respect to the basepoint $j=\left(0_{n-1}, 1\right) \in \mathbb{H}^{n}$. Up to a scaling, $\nu_{j}$ is the weak-limit as $t \rightarrow \delta^{+}$of the family of measures

$$
\nu_{j}(t):=\frac{1}{\sum_{\gamma \in \Gamma} e^{-t d(j, \gamma j)}} \sum_{\gamma \in \Gamma} e^{-t d(j, \gamma j)} \delta_{\gamma(j)}
$$

where $\delta_{\gamma(j)}$ is the dirac measure at $\gamma(j)$.

We consider the Hilbert space $L^{2}(\Gamma \backslash G)$ where the inner product $\left\langle\psi_{1}, \psi_{2}\right\rangle$ is given by

$$
\left\langle\psi_{1}, \psi_{2}\right\rangle=\int_{\Gamma \backslash G} \psi_{1}(g) \overline{\psi_{2}(g)} d g
$$

where $d g$ denotes a $G$-invariant measure on $\Gamma \backslash G$. As $d g$ is an invariant measure, the action of $G$ on $L^{2}(\Gamma \backslash G)$ by right translations gives rise to a unitary representation.

Since the complementary series representations exhaust the nontempered spectrum of $\hat{G}$ (cf. [19, Thm. 16. 2-3]), we deduce the following from [36] and [22]: recall the notation $\mathcal{H}_{s}$ of the complementary series representation of $G$ on which $\mathcal{C}$ acts by $s(s-n+1)$.

Theorem 3.1. There exist

$$
\alpha_{0}=\delta(\delta-n+1)>\alpha_{1} \geq \cdots \geq \alpha_{k}>-(n-1)^{2} / 4
$$

such that

$$
L^{2}(\Gamma \backslash G)=\mathcal{H}_{s_{0}=\delta} \oplus \cdots \oplus \mathcal{H}_{s_{k}} \oplus \mathcal{W}
$$


where $(n-1) / 2<s_{i}<(n-1)$ is given by the equation $\alpha_{i}=s_{i}\left(s_{i}-n+1\right)$ and $\mathcal{W}$ lies in the tempered spectrum of $\hat{G}$.

We set $V:=\mathcal{H}_{\delta}$. The base eigenfunction $\phi_{0} \in V^{K}$ for the Laplacian $\Delta$ can be explicitly written as the integral of the Poisson kernel against $\nu_{j}([36]):$

$$
\phi_{0}\left(n_{x} a_{y}\right)=\int_{u \in \mathbb{R}^{n}} \hat{\phi}_{u}(x, y) d \nu_{j}(u)
$$

where $\hat{\phi}_{u}(x, y):=\left(\frac{\left(|u|^{2}+1\right) y}{|x-u|^{2}+y^{2}}\right)^{\delta}$. We normalize $\nu_{j}$ so that $\left\|\phi_{0}\right\|_{2}=1$.

For $n=2$, we set

$$
\psi_{\ell}= \begin{cases}\mathcal{R}^{\ell}\left(\phi_{0}\right) & \text { if } \ell \geq 0 \\ \overline{\mathcal{R}}^{|\ell|}\left(\phi_{0}\right) & \text { if } \ell<0\end{cases}
$$

Sine $\overline{\mathcal{R}}$ is the complex conjugate of $\mathcal{R}, \psi_{-\ell}=\overline{\psi_{\ell}}$. Therefore for $n=2$, it suffices to describe $\psi_{\ell}$ for $\ell \geq 0$.

For $n=3$, we define $\psi_{\ell}$ recursively:

$$
\psi_{0}:=\phi_{0}, \quad \text { and } \quad \psi_{\ell}=\mathcal{Z}_{\ell}\left(\psi_{\ell-1}\right) \quad \text { for each } \ell \geq 1 \text {. }
$$

Definition 3.2. Let $n=2,3$. For each $\ell \in \hat{K}$, define the unit vector in $V_{\ell}^{M}$ by:

$$
\phi_{\ell}:=\frac{\psi_{\ell}}{\left\|\psi_{\ell}\right\|_{2}} \in C^{\infty}(\Gamma \backslash G)^{M} \cap L^{2}(\Gamma \backslash G) .
$$

We have by Corollary 2.14 ,

$$
V^{M}=\oplus_{\ell \in \hat{K}} \mathbb{C} \phi_{\ell}
$$

The rest of this section is devoted to obtaining pointwise bounds for these base eigenfunctions $\phi_{\ell}$ 's in terms of $\phi_{0}$.

\subsection{Base eigenfunctions for $G=\mathrm{PSL}_{2}(\mathbb{R})$.}

Theorem 3.3. Let $G=\mathrm{PSL}_{2}(\mathbb{R})$. For $\ell \in \mathbb{Z}_{\geq 0}$,

$$
\phi_{\ell}\left(n_{x} a_{y}\right)=\frac{\sqrt{\Gamma(1-\delta) \Gamma(\ell+\delta)}}{\sqrt{\Gamma(\delta) \Gamma(\ell+1-\delta)}} \cdot \int_{\mathbb{R}} \hat{\phi}_{u}(x, y)\left(\frac{(x-u)-i y}{(x-u)+i y}\right)^{\ell} d \nu_{j}(u) .
$$

In particular,

$$
\left|\phi_{ \pm \ell}\left(n_{x} a_{y}\right)\right| \ll \phi_{0}\left(n_{x} a_{y}\right)
$$

with implied constant independent of $\ell$.

Proof. We have

$$
K=\left\{k_{\theta}=\left(\begin{array}{cc}
\cos \theta & \sin \theta \\
-\sin \theta & \cos \theta
\end{array}\right): \theta \in[0, \pi)\right\} .
$$


The raising operator $\mathcal{R}$ in the Iwasawa coordinates $n_{x} a_{y} k_{\theta}$ can be written as

$$
\mathcal{R}=e^{2 i \theta}\left(i y \frac{\partial}{\partial x}+y \frac{\partial}{\partial y}+\frac{1}{2 i} \frac{\partial}{\partial \theta}\right)
$$

(see [1]). Since $\left\|\psi_{\ell}\right\|$ is given in $(2.6)$, it suffices to show that

$$
\psi_{\ell}\left(n_{x} a_{y} k_{\theta}\right)=\frac{e^{2 \ell i \theta} \Gamma(\delta+\ell)}{\Gamma(\delta)} \int_{\mathbb{R}} \hat{\phi}_{u}(x, y)\left(\frac{(x-u)-i y}{(x-u)+i y}\right)^{\ell} d \nu_{j}(u) .
$$

The case $\ell=0$ is clear. To use an induction, we assume that (3.4) holds for $\ell$. We compute

$$
\begin{aligned}
& \left(i y \frac{\partial}{\partial x}+y \frac{\partial}{\partial y}\right)\left(\hat{\phi}_{u}(x, y) \cdot\left(\frac{(x-u)-i y}{(x-u)+i y}\right)^{\ell}\right) \\
& =\delta \cdot \hat{\phi}_{u}(x, y) \cdot\left(\frac{(x-u)-i y}{(x-u)+i y}\right)^{\ell+1}+\ell \cdot \hat{\phi}_{u}(x, y) \cdot\left(\frac{(x-u)-i y}{(x-u)+i y}\right)^{\ell} \cdot\left(\frac{-2 i y}{(x-u)+i y}\right)
\end{aligned}
$$

and

$$
\begin{aligned}
\frac{1}{2 i} \frac{\partial}{\partial \theta}\left(\mathcal{R}^{\ell} \phi_{0}\right)\left(n_{x} a_{y} k_{\theta}\right) & \\
= & \frac{\Gamma(\delta+\ell)}{\Gamma(\delta)} \cdot \ell \cdot e^{2 i \ell \theta} \int_{\mathbb{R}} \hat{\phi}_{u}(x, y)\left(\frac{(x-u)-i y}{(x-u)+i y}\right)^{\ell} d \nu_{j}(u) .
\end{aligned}
$$

Hence

$$
\left(\mathcal{R}^{\ell+1} \phi_{0}\right)\left(n_{x} a_{y} k_{\theta}\right)=\frac{e^{2 i(\ell+1) \theta} \Gamma(\delta+\ell+1)}{\Gamma(\delta)} \int_{\mathbb{R}} \hat{\phi}_{u}(x, y)\left(\frac{(x-u)-i y}{(x-u)+i y}\right)^{\ell+1} d \nu_{j}(u) .
$$

Hence (3.4) holds for $\ell+1$, finishing the proof.

3.2. Base eigenfunctions for $G=\mathrm{PSL}_{2}(\mathbb{C})$. The case of $n=3$ involves more complicated computations and it turns out that $\phi_{\ell}$ 's are not uniformly bounded by $\phi_{0}$, but grow polynomially as $\ell \rightarrow \infty$ (see Theorem 3.8).

We parametrize elements of $K=\mathrm{PSU}(2)$ as

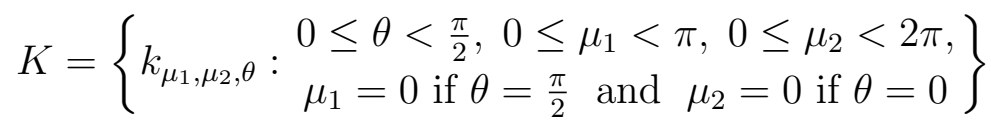

where

$$
k_{\mu_{1}, \mu_{2}, \theta}:=\left(\begin{array}{cc}
e^{i \mu_{1}} \cos \theta & e^{i \mu_{2}} \sin \theta \\
-e^{-i \mu_{2}} \sin \theta & e^{-i \mu_{1}} \cos \theta
\end{array}\right)
$$


Using the coordinates $\left(x_{1}, x_{2}, y, \mu_{1}, \mu_{2}, \theta\right)$, the element $\tilde{H}$ defined in (2.3) is given by

$$
\begin{aligned}
\tilde{H}=-\cos \left(\mu_{1}+\mu_{2}\right) \sin (2 \theta) y \frac{\partial}{\partial x_{1}}- & \sin \left(\mu_{1}+\mu_{2}\right) \sin (2 \theta) y \frac{\partial}{\partial x_{2}} \\
& +\cos (2 \theta) y \frac{\partial}{\partial y}+\frac{\sin (2 \theta)}{2} \frac{\partial}{\partial \theta} .
\end{aligned}
$$

Recall

$$
\hat{\phi}_{u}(x, y)=\left(\frac{\left(|u|^{2}+1\right) y}{|x-u|^{2}+y^{2}}\right)^{\delta} .
$$

Setting $\hat{\phi}_{u}\left(n_{x} a_{y} k\right)=\hat{\phi}_{u}(x, y, k):=\hat{\phi}_{u}(x, y)$, we may regard $\hat{\phi}_{u}$ as a function on $G$ and define functions $\hat{\phi}_{u}^{(\ell)}$ on $G$ by the recursive formula: for each $\ell \geq 1$,

$$
\hat{\phi}_{u}^{(\ell)}:=(-2 \ell+1) \tilde{H}\left(\hat{\phi}_{u}^{(\ell-1)}\right)+(\ell-1)^{2}(\delta(2-\delta)+\ell(\ell-2)) \hat{\phi}_{u}^{(\ell-2)}
$$

(as before, the terms which are not defined are understood as 0 ).

Lemma 3.6. For each $\ell \geq 0$, and $n_{x} a_{y} \in N A$, we have

$$
\psi_{\ell}\left(n_{x} a_{y}\right)=\int_{u \in \mathbb{R}^{2}} \hat{\phi}_{u}^{(\ell)}(x, y, e) d \nu_{j}(u)
$$

Proof. By the recursive formula for $\mathcal{Z}_{\ell}$ given in Theorem 2.11, for $\ell \geq 1$, we have

$$
\psi_{\ell+1}=\mathcal{Z}_{\ell}\left(\psi_{\ell-1}\right)=a_{\ell} \tilde{H}\left(\psi_{\ell}\right)+b_{\ell} \psi_{\ell-1}
$$

where $a_{\ell}=-2 \ell+1$ and $b_{\ell}=(\ell-1)^{2}(\delta(2-\delta)+\ell(\ell-2))$.

We prove the claim by induction. The case of $\ell=0$ is by definition of $\psi_{0}$. Suppose the claim holds for all $j \leq \ell-1$. Observe that all the terms in 3.5 for $\tilde{H}$ except for the term $y \frac{\partial}{\partial y}$ have $\sin (2 \theta)$ and hence vanish when $\theta=0$. Therefore

$$
\begin{aligned}
\psi_{\ell}\left(n_{x} a_{y}\right) & =a_{\ell} y \frac{\partial \psi_{\ell-1}}{\partial y}\left(n_{x} a_{y}\right)+b_{\ell} \psi_{\ell-2}\left(n_{x} a_{y}\right) \\
& =a_{\ell} y \frac{\partial}{\partial y} \int_{u \in \mathbb{R}^{2}} \hat{\phi}_{u}^{(\ell-1)}(x, y) d \nu_{j}(u)+b_{\ell} \int_{u \in \mathbb{R}^{2}} \hat{\phi}_{u}^{(\ell-2)}(x, y) d \nu_{j}(u) \\
& =\int_{u \in \mathbb{R}^{2}}\left(a_{\ell}\left(\tilde{H} \hat{\phi}_{u}^{(\ell-1)}\right)(x, y, e)+b_{\ell} \hat{\phi}_{u}^{(\ell-2)}(x, y, e)\right) d \nu_{j}(u) \\
& =\int_{u \in \mathbb{R}^{2}} \hat{\phi}_{u}^{(\ell)}(x, y, e) d \nu_{j}(u) .
\end{aligned}
$$

It turns out that the Legendre polynomials appear in the formula for $\phi_{\ell}$ : let us denote by $\mathbf{P}_{\ell}(t)$ the Legendre polynomial of degree $\ell$. It is defined by the recursive relation: $\mathbf{P}_{0}(t)=1, \mathbf{P}_{1}(t)=t$ and

$$
\ell \cdot \mathbf{P}_{\ell}(t)=(2 \ell-1) t \mathbf{P}_{\ell-1}(t)-(\ell-1) \mathbf{P}_{\ell-2}(t) .
$$


Theorem 3.8. Let $\ell \geq 0$. For any $n_{x} a_{y} \in N A$, we have

$$
\phi_{\ell}\left(n_{x} a_{y}\right)=\sqrt{2 \ell+1} \cdot \frac{\sqrt{\Gamma(2-\delta) \Gamma(\ell+\delta)}}{\sqrt{\Gamma(\delta) \Gamma(\ell+2-\delta)}} \cdot \int_{u \in \mathbb{R}^{2}} \hat{\phi}_{u}(x, y) \cdot \mathbf{P}_{\ell}\left(\frac{y^{2}-|x-u|^{2}}{y^{2}+|x-u|^{2}}\right) d \nu_{j}(u)
$$

In particular,

$$
\left|\phi_{\ell}\left(n_{x} a_{y}\right)\right| \ll \sqrt{\ell+1} \cdot \phi_{0}\left(n_{x} a_{y}\right)
$$

with the implied constant independent of $\ell$.

Proof. Since $\left\|\psi_{\ell}\right\|$ 's have been computed in Lemma 2.13, it suffices to show that

$$
\psi_{\ell}\left(n_{x} a_{y}\right)=\ell ! \frac{\Gamma(\delta+\ell)}{\Gamma(\delta)} \int_{u \in \mathbb{R}^{2}} \hat{\phi}_{u}(x, y) \cdot \mathbf{P}_{\ell}\left(\frac{y^{2}-|x-u|^{2}}{y^{2}+|x-u|^{2}}\right) d \nu_{j}(u) .
$$

Since $\psi_{\ell}\left(n_{x} a_{y}\right)=\int_{u \in \mathbb{R}^{2}} \hat{\phi}_{u}^{(\ell)}(x, y, e) d \nu_{j}(u)$ by Lemma 3.6. 3.9 follows if we show:

$$
\hat{\phi}_{u}^{(\ell)}(x, y, e)=\ell ! \frac{\Gamma(\delta+\ell)}{\Gamma(\delta)} \cdot \hat{\phi}_{u}(x, y) \cdot \mathbf{P}_{\ell}\left(\frac{y^{2}-|x-u|^{2}}{y^{2}+|x-u|^{2}}\right) .
$$

Put $B(x, y, u)=\frac{y^{2}-|x-u|^{2}}{y^{2}+|x-u|^{2}}$. Since

$$
y \frac{\partial}{\partial y} \hat{\phi}_{u}(x, y)=-\delta \cdot \hat{\phi}_{u}(x, y) \cdot B(x, y, u) \text { and } y \frac{\partial}{\partial y} B=-B^{2}+1,
$$

we have

$$
\begin{aligned}
& \hat{\phi}_{u}^{(1)}(x, y, e)=a_{1}\left(y \frac{\partial}{\partial y} \hat{\phi}_{u}\right)(x, y, e) \\
& =-\delta \hat{\phi}_{u}(x, y) \cdot a_{1} B=\delta \hat{\phi}_{u}(x, y) \mathbf{P}_{1}(B)
\end{aligned}
$$

proving the claim for $\ell=1$. For the induction, we assume that for all $j \leq \ell-1, \hat{\phi}_{u}^{(j)}(x, y, e)=d_{j} \hat{\phi}_{u}(x, y) j ! \cdot \mathbf{P}_{j}(B)$ where $d_{\ell}:=\prod_{j=1}^{\ell}(\delta+j-1)$. Then

$$
\begin{aligned}
& \hat{\phi}_{u}^{(\ell)}(x, y, e)=a_{\ell} \cdot\left(y \frac{\partial}{\partial y} \hat{\phi}_{u}^{(\ell-1)}\right)(x, y, e)+b_{\ell} \cdot \hat{\phi}_{u}^{(\ell-2)}(x, y, e)= \\
& a_{\ell} d_{\ell-1} \cdot(\ell-1) ! \cdot y \frac{\partial}{\partial y}\left(\hat{\phi}_{u}(x, y) \mathbf{P}_{\ell-1}(B)\right)+b_{\ell} d_{\ell-2} \cdot \hat{\phi}_{u}(x, y) \cdot(\ell-2) ! \cdot \mathbf{P}_{\ell-2}(B) .
\end{aligned}
$$

Since $b_{\ell}=(\ell-1)^{2}(-\delta+\ell)(\delta+\ell-2)$, we have

$$
d_{\ell-2} b_{\ell}=(\ell-1)^{2} d_{\ell-1}(-\delta+\ell) .
$$


Therefore

$$
\begin{aligned}
& \hat{\phi}_{u}^{(\ell)}(x, y, e) \\
& =d_{\ell-1}\left\{-a_{\ell} \delta B(\ell-1) ! \mathbf{P}_{\ell-1}(B)-a_{\ell}\left(B^{2}-1\right)(\ell-1) ! \mathbf{P}_{\ell-1}^{\prime}(B)\right. \\
& \left.+(\ell-1)^{2}(-\delta+\ell)(\ell-2) ! \mathbf{P}_{\ell-2}(B)\right\} \hat{\phi}_{u}(x, y) \\
& =d_{\ell-1}\left\{-a_{\ell} \delta B(\ell-1) ! \mathbf{P}_{\ell-1}(B)-\delta(\ell-1)^{2}(\ell-2) ! \mathbf{P}_{\ell-2}(B)\right. \\
& \left.-a_{\ell}\left(B^{2}-1\right)(\ell-1) ! \mathbf{P}_{\ell-1}^{\prime}(B)+\ell(\ell-1)^{2}(\ell-2) ! \mathbf{P}_{\ell-2}(B)\right\} \hat{\phi}_{u}(x, y) .
\end{aligned}
$$

Since

$(\ell-1)(\ell-1) ! \mathbf{P}_{\ell}(B)=-a_{\ell}\left(B^{2}-1\right)(\ell-1) ! \mathbf{P}_{\ell-1}^{\prime}(B)+(\ell-1)^{2} \ell(\ell-2) ! \mathbf{P}_{\ell-2}(B)$,

we have

$$
\begin{aligned}
\hat{\phi}_{u}^{(\ell)}(x, y, e) & =d_{\ell-1} \hat{\phi}_{u}(x, y) \cdot(\delta+\ell-1) \ell ! \mathbf{P}_{\ell}(B) \\
& =d_{\ell} \cdot \hat{\phi}_{u}(x, y) \cdot \ell ! \mathbf{P}_{\ell}(B) .
\end{aligned}
$$

This proves $(3.10)$, in view of the relation $\Gamma(t+1)=t \Gamma(t)$ and $d_{\ell}=$ $\frac{\Gamma(\delta+\ell)}{\Gamma(\delta)}$. Hence the first claim of the theorem is proved.

Since $\left|\mathbf{P}_{\ell}(t)\right| \leq 1$ for $|t| \leq 1$ (see p. 987 of [17]), the second claim follows from the first claim.

We do not know whether for all $n_{x} a_{y} k \in G,\left|\phi_{\ell}\left(n_{x} a_{y} k\right)\right|$ is uniformly bounded by $\ell^{N} \phi_{0}\left(n_{x} a_{y}\right)$ for some $N>0$. However for our purpose it suffices to prove the following bound:

Theorem 3.11. For each $\ell \geq 0$, there exists a constant $C_{\ell}>0$ such that for any $n_{x} a_{y} k \in N A K$,

$$
\left|\phi_{\ell}\left(n_{x} a_{y} k\right)\right| \leq C_{\ell} \cdot \phi_{0}\left(n_{x} a_{y}\right) .
$$

Proof. We use notations from the proof of Theorem 3.8. We compute

$$
y \frac{\partial}{\partial x_{1}} \hat{\phi}_{u}=\delta \cdot \hat{\phi}_{u} \cdot \frac{-2 y\left(x_{1}-u_{1}\right)}{|x-u|^{2}+y^{2}}, \quad y \frac{\partial}{\partial x_{2}} \hat{\phi}_{u}=\delta \cdot \hat{\phi}_{u} \cdot \frac{-2 y\left(x_{2}-u_{2}\right)}{|x-u|^{2}+y^{2}}
$$

and

$$
y \frac{\partial}{\partial y} \hat{\phi}_{u}=\delta \cdot \hat{\phi}_{u} \cdot \frac{|x-u|^{2}-y^{2}}{|x-u|^{2}+y^{2}} .
$$

Let

$$
A_{1}(x, y, u)=\frac{-2 y\left(x_{1}-u_{1}\right)}{|x-u|^{2}+y^{2}}, \quad A_{2}(x, y, u)=\frac{-2 y\left(x_{2}-u_{2}\right)}{|x-u|^{2}+y^{2}}
$$

and

$$
B(x, y, u)=\frac{|x-u|^{2}-y^{2}}{|x-u|^{2}+y^{2}} .
$$

For $\mu=\left(\mu_{1}, \mu_{2}\right)$ and $0 \leq \theta<\pi$, define

$\Phi_{1}(\mu, \theta):=-\sin (2 \theta) \cos \left(\mu_{1}+\mu_{2}\right)$, and $\Phi_{2}(\mu, \theta):=-\sin (2 \theta) \sin \left(\mu_{1}+\mu_{2}\right)$ 
and $\Psi(\theta):=\cos (2 \theta)$ so that by 3.5$)$,

$$
\tilde{H}=\Phi_{1} y \frac{\partial}{\partial x_{1}}+\Phi_{2} y \frac{\partial}{\partial x_{2}}+\Psi y \frac{\partial}{\partial y}+\frac{\sin (2 \theta)}{2} \frac{\partial}{\partial \theta} .
$$

Hence

$$
\tilde{H} \hat{\phi}_{u}=\delta \cdot \hat{\phi}_{u} \cdot\left(\Phi_{1} A_{1}+\Phi_{2} A_{2}+\Psi B\right)
$$

SO

$$
\hat{\phi}_{u}^{(1)}=\hat{\phi}_{u} \cdot a_{1} \delta \cdot\left(\Phi_{1} A_{1}+\Phi_{2} A_{2}+\Psi B\right) .
$$

We compute

$$
\begin{aligned}
& y \frac{\partial}{\partial x_{1}} A_{1}=A_{1}^{2}+B-1, \quad y \frac{\partial}{\partial x_{2}} A_{1}=A_{1} A_{2}+B-1, \quad y \frac{\partial}{\partial y} A_{1}=A_{1} B \\
& y \frac{\partial}{\partial x_{1}} A_{2}=A_{1} A_{2}+B-1, \quad y \frac{\partial}{\partial x_{2}} A_{2}=A_{2}^{2}+B-1, \quad y \frac{\partial}{\partial y} A_{2}=A_{2} B
\end{aligned}
$$

and

$$
y \frac{\partial}{\partial x_{1}} B=A_{1}(B-1), \quad y \frac{\partial}{\partial x_{2}} B=A_{2}(B-1), \quad y \frac{\partial}{\partial y} B=(B-1)(B+1) .
$$

We also compute:

$$
\tilde{H}\left(\Phi_{1}\right)=\Phi_{1} \Psi, \quad \tilde{H}\left(\Phi_{2}\right)=\Phi_{2} \Psi
$$

and

$$
\tilde{H}(\Psi)=-\Phi_{1}^{2}-\Phi_{2}^{2}=-1+\Psi^{2} .
$$

It follows that $\hat{\phi}_{u}^{(\ell)}=\hat{\phi}_{u} \cdot p_{\ell}\left(\Phi_{1}, \Phi_{2}, A_{1}, A_{2}, \Psi, B\right)$ where $p_{\ell}$ is a polynomial in $\Phi_{1}, \Phi_{2}, A_{1}, A_{2}, \Psi$ and $B$, whose coefficients are given by monomials in $\left\{ \pm 1, a_{1}, \ldots, a_{\ell}, b_{1}, \ldots, b_{\ell}, \delta\right\}$. Since the absolute values of $\Phi_{1}, \Phi_{2}, \Psi, A_{1}, A_{2}$ and $B$ are all bounded above by 1 , we deduce

$$
\left|\hat{\phi}_{u}^{(\ell)}(x, y, k)\right| \leq C_{\ell} \cdot \hat{\phi}_{u}(x, y) \text {. }
$$

for some constant $C_{\ell}>0$ independent of $x, y, k$. Since

$$
\psi_{\ell}\left(n_{x} a_{y} k\right)=\int_{u \in \Lambda(\Gamma)} \hat{\phi}_{u}^{(\ell)}(x, y, k) d \nu_{j}(u),
$$

the claim follows.

\section{Horospherical AVERAGe of $\phi_{\ell}$}

We let the notations $G=N A K, \Gamma, \delta, \phi_{0}, \phi_{\ell}$, etc., be as in Section 3. We assume that $\Gamma$ is geometrically finite with $(n-1) / 2<\delta<$ $(n-1)$ and that $\Gamma \backslash \Gamma N$ is closed in $\Gamma \backslash G$ in the whole section. The main goal of this section is to compute the horospherical average of $\phi_{\ell}$ over $(\Gamma \cap N) \backslash N$ (Theorem 4.12). Consider $\mathbb{H}^{n}$ for $n=2$ or 3 according as $G=\mathrm{PSL}_{2}(\mathbb{R})$ or $\mathrm{PSL}_{2}(\mathbb{C})$. We let $X_{0} \in \mathrm{T}^{1}\left(\mathbb{H}^{n}\right)$ be the upward normal vector based at $j=\left(0_{n-1}, 1\right) \in \mathbb{H}^{n}$. The map $g \mapsto g\left(X_{0}\right)$ induces the identification of $G / M$ with $\mathrm{T}^{1}\left(\mathbb{H}^{n}\right)$. The horosphere in $\mathrm{T}^{1}\left(\mathbb{H}^{n}\right)$ 
corresponding to $N M / M$ consists of the upward normal vectors on the horizontal plane containing $j$, and hence based at $\infty \in \partial\left(\mathbb{H}^{n}\right)$.

The assumption that $\Gamma \backslash \Gamma N$ is closed is equivalent to saying that either $\infty \notin \Lambda(\Gamma)$ or $\infty$ is a parabolic fixed point of $\Gamma$ [10]. Recall $\xi \in \Lambda(\Gamma)$ is a parabolic fixed point if it is a unique fixed point in $\partial\left(\mathbb{H}^{n}\right)$ of an element of $\Gamma$. One of the important features of a geometrically finite group $\Gamma$ is that any parabolic fixed point $\xi$ of $\Gamma$ is bounded, meaning that the stabilizer $\operatorname{Stab}_{\Gamma}(\xi)$ acts cocompactly on $\Lambda(\Gamma)-\{\xi\}$ [8].

Therefore our assumption on the closedness of $\Gamma \backslash \Gamma N$ implies the following:

Lemma 4.1. $N \cap \Gamma$ acts cocompactly on $\Lambda(\Gamma)-\{\infty\}$.

As mentioned in the introduction, the rank of $\infty$ is the rank of $\Gamma \cap N$ as a free abelian group.

Definition 4.2. Given $\psi \in C(\Gamma \backslash G)^{M}$, define $\psi^{N} \in C(\Gamma \backslash G)^{M}$ by

$$
\psi^{N}(g):=\int_{n_{x} \in(N \cap \Gamma) \backslash N} \psi\left(n_{x} g\right) d x
$$

where $d x$ denotes the Lebesgue measure on $\mathbb{R}^{n}$, provided the integral converges.

Proposition 4.3. There exists $c_{n}(0)>0$ such that for all $y>0$,

$$
\phi_{0}^{N}\left(a_{y}\right)=c_{n}(0) \cdot y^{n-1-\delta} .
$$

Proof. In [20], it was shown that $\phi_{0}^{N}\left(a_{y}\right)$ converges absolutely and that there are constants $c_{n}(0)>0$ and $d_{n}(0) \in \mathbb{R}$ such that for all $y>0$

$$
\phi_{0}^{N}\left(a_{y}\right)=c_{n}(0) y^{n-1-\delta}+d_{n}(0) y^{\delta} .
$$

Since $\phi_{0}>0$ and (4.4) holds for all $y>0$, it follows that $d_{n}(0) \geq 0$. We claim that $d_{n}(0)=0$.

When $\infty \notin \Lambda(\Gamma), \Lambda(\Gamma)$ is a bounded subset of $\mathbb{R}^{n-1}$ and we can show by direct computations:

$$
\phi_{0}^{N}\left(a_{y}\right)= \begin{cases}\frac{\sqrt{\pi} \Gamma\left(\delta-\frac{1}{2}\right)}{\Gamma(\delta)} \cdot \int_{u \in \Lambda(\Gamma)}\left(|u|^{2}+1\right)^{\delta} d \nu_{j}(u) \cdot y^{1-\delta} & \text { if } n=2 \\ \frac{\pi}{\delta-1} \cdot \int_{u \in \Lambda(\Gamma)}\left(|u|^{2}+1\right)^{\delta} d \nu_{j}(u) \cdot y^{2-\delta} & \text { if } n=3\end{cases}
$$

(see [20]).

Now suppose $\infty \notin \Lambda(\Gamma)$. As $\Gamma$ is geometrically finite, $\Gamma$ admits a polyhedron fundamental domain $\mathcal{F}$ in $\mathbb{H}^{n}$ such that $F_{0} \times\left[Y_{0}, \infty\right)$ injects to $\mathcal{F}$ for some $Y_{0} \gg 1$ where $F_{0}$ is a fundamental domain in $\mathbb{R}^{n-1}$ for $N \cap \Gamma$. Let $B_{t}=\left\{x \in F_{0}:|x|<t\right\}$ for $t>1$. 
When $\infty$ is a bounded parabolic fixed point of rank $n-1$, take $t_{0}$ so that $B_{t_{0}}=F_{0}$, which is possible since $F_{0}$ is bounded in this case. Then

$$
\int_{x \in B_{t_{0}}} \phi_{0}\left(n_{x} a_{y}\right) d x \geq \frac{d_{n}(0)}{2} y^{\delta} .
$$

Therefore using the Cauchy-Schwartz inequality, we have

$$
\begin{aligned}
\left\|\phi_{0}\right\|_{2}^{2} & \geq \int_{Y_{0}}^{\infty} \int_{B_{t_{0}}} \phi_{0}\left(n_{x} a_{y}\right)^{2} y^{-n} d x d y \\
& \geq \frac{1}{\operatorname{vol}\left(B_{t_{0}}\right)} \int_{Y_{0}}^{\infty}\left(\int_{B_{t_{0}}} \phi_{0}\left(n_{x} a_{y}\right) d x\right)^{2} y^{-n} d y \\
& \geq \frac{d_{n}(0)^{2}}{4 \operatorname{vol}\left(B_{t_{0}}\right)} \int_{Y_{0}}^{\infty} y^{2 \delta-n} d y .
\end{aligned}
$$

Since $\delta>(n-1) / 2,\left\|\phi_{0}\right\|_{2}=\infty$ unless $d_{n}(0) \neq 0$. Therefore $d_{n}(0)=0$.

The remaining case is when $n=3$ and $\infty$ is a bounded parabolic fixed point of rank one. In this case, it was shown in the proof of [20, Prop. 4.6] that for all sufficiently large $t \gg 1$, there exists $b_{t} \rightarrow 0$ as $t \rightarrow \infty$ such that for all $y>0$,

$$
\int_{x \in F_{0}-B_{t}} \phi_{0}\left(n_{x} a_{y}\right) d x \leq b_{t} y^{\delta} .
$$

Therefore, if $d_{3}(0)$ were positive, then for some large $t_{0}>0$, we would have

$$
\int_{x \in B_{t_{0}}} \phi_{0}\left(n_{x} a_{y}\right) d x \geq \frac{d_{3}(0)}{2} y^{\delta} .
$$

By repeating the same argument as in the previous case, this leads to a contradiction.

Lemma 4.8. Let $y>0$ and $k \in K$. For each $1 \leq i \leq n-1$, we have

$$
\int_{(N \cap \Gamma) \backslash N} \frac{\partial}{\partial x_{i}} \phi_{\ell}\left(n_{x} a_{y} k\right) d x=0 .
$$

Proof. By Theorems 3.3 and 3.11, we have, for some $C_{\ell}>0$,

$$
\left|\phi_{\ell}\left(n_{x} a_{y} k\right)\right| \leq C_{\ell} \cdot \phi_{0}\left(n_{x} a_{y}\right) .
$$

Suppose $n=3$. If $\infty \notin \Lambda(\Gamma)$ and hence $N \cap \Gamma=\{e\},\left|\phi_{0}\left(n_{x} a_{y} k\right)\right| \rightarrow 0$ as $|x| \rightarrow \infty$. Hence, by (4.9), as $|x| \rightarrow \infty$,

$$
\left|\phi_{\ell}\left(n_{x} a_{y} k\right)\right| \rightarrow 0
$$


Therefore

$$
\begin{aligned}
& \int_{x_{1} \in \mathbb{R}} \frac{\partial}{\partial x_{1}} \phi_{\ell}\left(n_{x} a_{y} k\right) d x_{1} \\
& =\lim _{t \rightarrow \infty} \int_{-t}^{t} \frac{\partial}{\partial x_{1}} \phi_{\ell}\left(n_{x} a_{y} k\right) d x_{1} \\
& =\lim _{t \rightarrow \infty}\left(\phi_{\ell}\left(n_{t+\sqrt{-1} x_{2}} a_{y} k\right)-\phi_{\ell}\left(n_{-t+\sqrt{-1} x_{2}} a_{y} k\right)\right)=0 .
\end{aligned}
$$

The other case of $i=2$ is symmetric to this one.

When $\infty$ is a bounded parabolic fixed point of rank one, we may assume without loss of generality that $N \cap \Gamma$ is generated by $n_{1}=$ $\left(\begin{array}{ll}1 & 1 \\ 0 & 1\end{array}\right)$, so that there exists a fundamental domain $F_{0}$ in $\mathbb{R}^{2}$ inside $\left\{\left(x_{1}, x_{2}\right): 0 \leq x_{1} \leq 1\right\}$. In this case, $\phi_{0}\left(n_{x} a_{y}\right) \rightarrow 0$ as $x_{2} \rightarrow \infty$ [37]. Hence $\left|\phi_{\ell}\left(n_{x} a_{y} k\right)\right| \rightarrow 0$, as $x_{2} \rightarrow \infty$. By a similar argument as above, this implies that

$$
\int_{x_{2} \in \mathbb{R}} \frac{\partial}{\partial x_{2}} \phi_{\ell}\left(n_{x} a_{y} k\right) d x_{2}=0 .
$$

On the other hand, by the $n_{1} \in N \cap \Gamma$-invariance of $\phi_{\ell}$,

$$
\int_{x_{1} \in[0,1]} \frac{\partial}{\partial x_{1}} \phi_{\ell}\left(n_{x} a_{y} k\right) d x_{1}=\phi_{\ell}\left(n_{1} n_{\sqrt{-1} x_{2}} a_{y} k\right)-\phi_{\ell}\left(n_{\sqrt{-1} x_{2}} a_{y} k\right)=0 .
$$

If $\infty$ has rank 2, we may assume that $N \cap \Gamma$ is generated by $n_{1}$ and $n_{\sqrt{-1}}$. Then the the claim follows from Green's theorem and the invariance of $\phi_{\ell}$ by $N \cap \Gamma$ as in the last argument. The case $n=2$ can be shown similarly.

For $\ell \geq 0$, define

$$
M_{\ell}(\theta):=\ell ! \cdot \mathbf{P}_{\ell}(-\cos 2 \theta) .
$$

Then $M_{0}(\theta)=1$ and the recursive relation 3.7 for $\mathbf{P}_{\ell}$ 's implies:

Lemma 4.11. (1) For $\ell \geq 1$,

$$
M_{\ell}(\theta)=(-2 \ell+1) \cos (2 \theta) M_{\ell-1}(\theta)-(\ell-1)^{2} M_{\ell-2}(\theta) .
$$

(2) For each $\ell \geq 1$,

$$
\begin{aligned}
2(\ell+1) M_{\ell}(\theta) & =4(-2 \ell+1) \cos (2 \theta) M_{\ell-1}(\theta) \\
+ & (-2 \ell+1) \sin (2 \theta) M_{\ell-1}^{\prime}(\theta)+2(\ell-1)^{2}(\ell-2) M_{\ell-2}(\theta) .
\end{aligned}
$$

The following theorem implies in particular that the integral $\phi_{\ell}^{N}\left(a_{y}\right)$ converges, which is a priori unclear as $(N \cap \Gamma) \backslash N$ is not compact in general. 
Theorem 4.12. For $\ell \geq 0$, we have

$$
\phi_{\ell}^{N}\left(a_{y}\right)=(-1)^{(n-2) \ell} \cdot c_{n}(0) \cdot \frac{\sqrt{\Gamma(n-1-\delta) \Gamma(\ell+\delta)}}{\sqrt{\Gamma(\delta) \Gamma(\ell+n-1-\delta)}} \cdot \sqrt{2(n-2) \ell+1} \cdot y^{n-1-\delta} .
$$

In particular,

$$
\left|\phi_{\ell}^{N}\left(a_{y}\right)\right| \ll \ell^{(n-2) / 2} \cdot y^{n-1-\delta}
$$

with the implied constant independent of $\ell \geq 1$.

Proof. By Theorems 3.3 and 3.8, we have

$$
\begin{aligned}
\left|\int_{(N \cap \Gamma) \backslash N} \phi_{\ell}\left(n_{x} a_{y}\right) d x\right| & \leq \int_{(N \cap \Gamma) \backslash N}\left|\phi_{\ell}\left(n_{x} a_{y}\right)\right| d x \\
& \ll \int_{(N \cap \Gamma) \backslash N} \phi_{0}\left(n_{x} a_{y}\right) d x .
\end{aligned}
$$

Hence by Proposition 4.3, the integral $\phi_{\ell}^{N}\left(a_{y}\right)$ converges absolutely. Let $n=2$. By (2.6), it suffices to show

$$
\psi_{\ell}^{N}\left(a_{y} k_{\theta}\right)=e^{2 \ell i \theta} c_{2}(0) \frac{\Gamma(\ell+1-\delta)}{\Gamma(1-\delta)} y^{1-\delta} .
$$

The case $\ell=0$ holds by Proposition 4.3. To use an induction, we assume 4.13 is true for $\ell$. Then applying Lemma 4.8,

$$
\begin{aligned}
\psi_{\ell+1}^{N}\left(a_{y} k_{\theta}\right)= & e^{2 i \theta} \int_{(N \cap \Gamma) \backslash N}\left(i y \frac{\partial}{\partial x}+y \frac{\partial}{\partial y} \frac{1}{2 i} \frac{\partial}{\partial \theta}\right) \psi_{\ell}\left(n_{x} a_{y} k_{\theta}\right) d x \\
& =e^{2 i \theta} \cdot\left(y \frac{\partial}{\partial y} \frac{1}{2 i} \frac{\partial}{\partial \theta}\right) \psi_{\ell}^{N}\left(a_{y} k_{\theta}\right) \\
& =e^{2 i \theta} \cdot\left(y \frac{\partial}{\partial y} \frac{1}{2 i} \frac{\partial}{\partial \theta}\right)\left(e^{2 \ell i \theta} c_{2}(0) \frac{\Gamma(\ell+1-\delta)}{\Gamma(1-\delta)} y^{1-\delta}\right) \\
& =e^{2(\ell+1) i \theta} \cdot\left(c_{2}(0) \cdot \frac{\Gamma(\ell+1-\delta)}{\Gamma(1-\delta)} \cdot((1-\delta)+\ell) y^{1-\delta}\right) \\
& =e^{2(\ell+1) i \theta} \cdot\left(c_{2}(0) \cdot \frac{\Gamma(\ell+1+(1-\delta))}{\Gamma(1-\delta)} y^{1-\delta}\right)
\end{aligned}
$$

since $z \Gamma(z)=\Gamma(z+1)$. This proves 4.13 for $\ell+1$.

Let $n=3$. Setting $q_{\ell}:=c_{3}(0) \prod_{j=1}^{\ell}(j+1-\delta)$, we claim that

$$
\psi_{\ell}^{N}\left(a_{y} k\right)=q_{\ell} \cdot y^{2-\delta} M_{\ell}(\theta)
$$

where $M_{\ell}(\theta)$ is defined as in 4.10. Set $k=k_{\mu_{1}, \mu_{2}, \theta}, a_{\ell}=-2 \ell+1$ and $b_{\ell}=(\ell-1)^{2}(\delta(2-\delta)+\ell(\ell-2))$ for simplicity.

Since $\phi_{0}$ is fixed by $K, E^{ \pm}\left(\phi_{0}\right)=0$. Hence

$$
\psi_{1}^{N}\left(a_{y} k\right)=a_{1} \int_{(N \cap \Gamma) \backslash N} \tilde{H}\left(\phi_{0}\right)\left(n a_{y} k\right) d n .
$$


By Proposition 4.3 and Lemma 4.8 , using $\frac{\partial}{\partial \theta} \phi_{0}\left(n_{x} a_{y} k\right)=0$ and (3.5), we have

$$
\begin{aligned}
\psi_{1}^{N}\left(a_{y} k\right) & =a_{1}\left(\cos (2 \theta) y \frac{\partial}{\partial y}+\frac{\sin (2 \theta)}{2} \frac{\partial}{\partial \theta}\right) \psi_{0}^{N}\left(a_{y} k\right) \\
& =a_{1} c_{3}(0) \cdot \cos (2 \theta)(2-\delta) y^{2-\delta}
\end{aligned}
$$

Hence (4.14) holds for $\ell=1$.

Assuming that (4.14) holds for $\ell$, we deduce using Lemma 4.11 that

$$
\begin{aligned}
& \psi_{\ell+1}^{N}\left(a_{y} k\right)=a_{\ell+1} q_{\ell} \tilde{H}\left(y^{2-\delta} M_{\ell}(\theta)\right)+b_{\ell+1} q_{\ell-1} y^{2-\delta} M_{\ell-1}(\theta) \\
& =(-2 \ell-1) q_{\ell}\left(\cos (2 \theta)(2-\delta) M_{\ell}(\theta)+\frac{\sin (2 \theta)}{2} M_{\ell}^{\prime}(\theta)\right) y^{2-\delta} \\
& \quad+\ell^{2}(\ell+1-\delta) q_{\ell-1}(\delta+\ell-1) y^{2-\delta} M_{\ell-1}(\theta) .
\end{aligned}
$$

By Lemma 4.11,

$$
M_{\ell+1}(\theta)=(-2 \ell-1) \cos (2 \theta) M_{\ell}(\theta)-\ell^{2} M_{\ell-1}(\theta)
$$

and

$$
\begin{aligned}
(\ell+2) M_{\ell+1}(\theta)=2(-2 \ell-1) \cos (2 \theta) M_{\ell}(\theta)+ & (-2 \ell-1) \frac{\sin (2 \theta)}{2} M_{\ell}^{\prime}(\theta) \\
& +\ell^{2}(\ell-1) M_{\ell-1}(\theta) .
\end{aligned}
$$

Therefore we have

$$
\psi_{\ell+1}^{N}\left(a_{y} k\right)=q_{\ell}(\ell+2-\delta) M_{\ell+1}(\theta) y^{2-\delta}=q_{\ell+1} M_{\ell+1}(\theta) y^{2-\delta},
$$

proving 4.14) for $\ell+1$.

By the formula of $\left\|\psi_{\ell}\right\|_{2}$ given in Lemma 2.13, and using $\left|\mathbf{P}_{\ell}(t)\right| \leq 1$ for all $t \in[-1,1]$ and $\mathbf{P}_{\ell}(-1)=(-1)^{\ell}$, we finish the proof for $n=3$.

\section{UNIFORM THICKENING FOR $\phi_{\ell}$ 'S}

We continue the notations $G=N A K, \Gamma$, $\phi_{\ell}$, etc. from section 4 . Assume that $\Gamma$ is geometrically finite with $\frac{n-1}{2}<\delta<n-1$ and that $\Gamma \backslash \Gamma N$ is closed in $\Gamma \backslash G$. In this section, we approximate the integral $\phi_{\ell}^{N}\left(a_{y}\right)$ as the inner product $\left\langle a_{y} \phi_{\ell}, \rho_{\eta, \epsilon}\right\rangle=\int_{\Gamma \backslash G} \phi_{\ell}\left(g a_{y}\right) \rho_{\eta, \epsilon}(g) d g$ for a suitable test function $\rho_{\eta, \epsilon}$. This step enables us to relate the horospherical average $\phi_{\ell}^{N}\left(a_{y}\right)$ with the spectral decomposition of $L^{2}(\Gamma \backslash G)$ stated in Theorem 3.1.

Fix a fundamental domain $F_{0}$ for $N \cap \Gamma$ in $\mathbb{R}^{n-1}$ and choose a compact fundamental domain $F_{\Lambda} \subset F_{0}$ for $(N \cap \Gamma) \backslash \Lambda(\Gamma)-\{\infty\}$ given by Lemma 4.1 .

Lemma 5.1. Fix $\ell \geq 0$. Suppose $\Gamma \backslash \Gamma N$ is not compact. For any open subset $J \subset F_{0}$ containing $\mathcal{F}_{\Lambda}$, we have for all $0<y<1$,

(1) $\int_{J^{c}} \phi_{0}\left(n_{x} a_{y}\right) d x \ll y^{\delta}$ with the implied constant independent of 
(2) $\left|\int_{F_{0}-J} \phi_{\ell}\left(n_{x} a_{y}\right) d x\right| \ll(\ell+1)^{(n-2) / 2} y^{\delta}$ with the implied constant independent of $\ell$.

Proof. Let $n=2$. Then the non-compactness assumption on $\Gamma \backslash \Gamma N$ implies that $\infty \notin \Lambda(\Gamma)$ and hence $\epsilon_{0}:=\inf _{x \notin J, u \in \Lambda(\Gamma)}|x-u|$ is positive. Then by the change of variable $w=\frac{x-u}{y}$, we have

$$
\int_{F_{0}-J} \phi_{0}\left(n_{x} a_{y}\right) d x \leq 2 y^{1-\delta} \int_{u \in \Lambda(\Gamma)}\left(u^{2}+1\right)^{\delta} d \nu_{i}(u) \cdot \int_{w=\epsilon_{0} / y}^{\infty}\left(\frac{1}{w^{2}+1}\right)^{\delta} d w .
$$

The latter integral can be evaluated explicitly as an incomplete Beta function which has known asymptotics:

$$
\int_{\epsilon_{0} / y}^{\infty}\left(\frac{1}{w^{2}+1}\right)^{\delta} d w=c \beta_{y^{2} / \epsilon_{0}^{2}}(\delta-1 / 2,1-\delta),
$$

where $\beta_{z}(\alpha, \beta) \ll z^{\alpha}$. Hence

$$
\int_{F_{0}-J} \phi_{0}\left(n_{x} a_{y}\right) d x \ll y^{1-\delta} \cdot y^{2(\delta-1 / 2)}=y^{\delta} .
$$

This proves (1) for $n=2$, and we refer to [20, Proposition 3.7] for $n=3$.

For the second claim, note that, by Theorem 3.8 ,

$$
\begin{aligned}
\left|\int_{x \in F_{0}-J} \phi_{\ell}\left(n_{x} a_{y}\right) d x\right| & \leq \int_{x \in F_{0}-J}\left|\phi_{\ell}\left(n_{x} a_{y}\right)\right| d x \\
& \ll(\ell+1)^{(n-2) / 2} \int_{F_{0}-J} \phi_{0}\left(n_{x} a_{y}\right) d x
\end{aligned}
$$

since $\phi_{0}$ is a positive function. Hence the claim (2) follows from (1).

For $m \geq 0$, the associated Legendre function $\mathbf{P}_{\ell}^{m}(x)$ is defined by the following:

$$
\mathbf{P}_{\ell}^{m}(x)=(-1)^{m}\left(1-x^{2}\right)^{m / 2} \frac{d^{m}}{d x^{m}}\left(\mathbf{P}_{\ell}(x)\right) .
$$

We set $\mathbf{P}_{\ell}^{-m}(x):=(-1)^{m} \frac{(\ell-m) !}{(\ell+m) !} \mathbf{P}_{\ell}^{m}(x)$.

Let $U_{\epsilon}$ denote the $\epsilon$-neighborhood of $e$ in $G$ for any $\epsilon>0$. The following lemma controls the Lipschitz constants of $\phi_{\ell}$ 's for the action of $K$ :

Lemma 5.3. Let $\ell \geq 0$. For all sufficiently small $\epsilon>0$,

$$
\phi_{\ell}\left(n_{x} a_{y} k\right)=\left(1+O\left((\ell+1)^{n-1} \epsilon\right)\right) \cdot \phi_{\ell}\left(n_{x} a_{y}\right)
$$

with the implied constant independent of $\ell, x, y>0$ and $k \in K_{\epsilon}:=$ $K \cap U_{\epsilon}$. 
Proof. If $n=2$, we have $\phi_{\ell}\left(n_{x} a_{y} k_{\theta}\right)=e^{2 \ell i \theta} \phi_{\ell}\left(n_{x} a_{y}\right)$, and hence the claim follows easily as $e^{2 \ell i \theta}=1+O((\ell+1) \theta)$ for all $\theta$ small.

Let $n=3$. We set $\mu=\mu_{1}+\mu_{2}$. The operator $\mathcal{C}_{K}$ acts on $M$-invariant functions as follows:

$$
-\mathcal{C}_{K} f(\mu, \theta)=\frac{1}{\sin ^{2} 2 \theta} \frac{\partial^{2}}{\partial \mu^{2}} f+\frac{1}{4 \sin 2 \theta} \frac{\partial}{\partial \theta}\left(\sin 2 \theta \frac{\partial}{\partial \theta} f\right) .
$$

Since $\mathcal{C}_{K}\left(\phi_{\ell}\right)=\ell(\ell+1) \phi_{\ell}$, it follows from the theory of spherical harmonics (cf. [35]) that

$$
\phi_{\ell}\left(n_{x} a_{y} k_{\mu_{1}, \mu_{2}, \theta}\right)=\sum_{m=-\ell}^{\ell} f_{\ell}^{m}(x, y) \cdot Y_{\ell}^{m}(\theta, \mu)
$$

where $f_{\ell, m} \in C^{\infty}\left(\mathbb{C} \times \mathbb{R}_{>0}\right)$ and

$$
Y_{\ell}^{m}(\theta, \mu)=\frac{\sqrt{(2 \ell+1)(\ell-m) !}}{\sqrt{4 \pi(\ell+m) !}} \cdot \mathbf{P}_{\ell}^{m}(\cos (2 \theta)) \cdot e^{i m \mu} .
$$

We have for $|\mu|<\epsilon, e^{i m \mu}=1+O(\ell \epsilon)$ as $|m| \leq \ell$. Also, from the properties of the associated Legendre functions, we deduce that for $|\theta|<\epsilon$ and $|m| \leq \ell$,

$$
\mathbf{P}_{\ell}^{m}(\cos 2 \theta)=(1+O(\ell \epsilon)) \mathbf{P}_{\ell}^{m}(1) .
$$

Therefore for $|\theta|<\epsilon$ and $|\mu|<\epsilon$,

$$
\mathbf{P}_{\ell}^{m}(\cos 2 \theta) \cdot e^{i m \mu}=\left(1+O\left(\ell^{2} \epsilon\right)\right) \mathbf{P}_{\ell}^{m}(1)
$$

and hence

$$
Y_{\ell}^{m}(\theta, \mu)=\left(1+O\left(\ell^{2} \epsilon\right)\right) Y_{\ell}^{m}(0,0) .
$$

It follows that for all $\ell \geq 1$,

$$
\begin{aligned}
\phi_{\ell}\left(n_{x} a_{y} k_{\mu_{1}, \mu_{2}, \theta}\right) & =\left(1+O\left(\ell^{2} \epsilon\right)\right) \sum_{m=-\ell}^{\ell} f_{\ell}^{m}(x, y) \cdot Y_{\ell}^{m}(0,0) \\
& =\left(1+O\left(\ell^{2} \epsilon\right)\right) \phi_{\ell}\left(n_{x} a_{y}\right) .
\end{aligned}
$$

Setting $N^{-}:=\left\{n_{x}^{-}:=\left(\begin{array}{ll}1 & 0 \\ x & 1\end{array}\right)\right\}$ where $x$ ranges over $\mathbb{R}$ (resp. $\mathbb{C}$ ) for $n=2$ (resp. $n=3$ ), the product map

$$
N \times A \times M \times N^{-} \rightarrow G
$$

is a diffeomorphism at a neighborhood of $e$. Let $d k$ be the invariant probability measure on $K$. For $g=n_{x} a_{y} k, d g=y^{-n} d x d y d k$ defines a Haar measure on $G$. Let $\nu$ be a smooth measure on $A M N^{-}$such that $d n_{x} \otimes d \nu=d g$. Fix a bounded open domain $J \subset F_{0}$ which contains $F_{\Lambda}$ and choose a compactly supported smooth function $0 \leq \eta \leq 1$ on $N$ 
with $\left.\eta\right|_{J}=1$. If $\infty$ is of rank $n-1$, then set $J=F_{0}$; hence $\eta=1$ on $F_{0}$.

Fix $\epsilon_{0}>0$ so that the multiplication map

$$
\operatorname{supp}(\eta) \times\left(U_{\epsilon_{0}} \cap A M N^{-}\right) \rightarrow \operatorname{supp}(\eta)\left(U_{\epsilon_{0}} \cap A M N^{-}\right) \subset \Gamma \backslash G
$$

is a bijection onto its image. For each $0<\epsilon<\epsilon_{0}$, let $0 \leq r_{\epsilon} \leq 1$ be a non-negative smooth function in $A M N^{-}$whose support is contained in $W_{\epsilon}:=\left(U_{\epsilon} \cap A\right) M\left(U_{\epsilon_{0}} \cap N^{-}\right)$and $\int_{W_{\epsilon}} r_{\epsilon} d \nu=1$.

We define the function $\rho_{\eta, \epsilon}$ on $\Gamma \backslash G$ as follows: for $g=n_{x} a_{y} m n_{x^{\prime}}^{-}$

$$
\rho_{\eta, \epsilon}(g)= \begin{cases}\eta\left(n_{x}\right) \cdot r_{\epsilon}\left(a_{y} m n_{x^{\prime}}^{-}\right) & \text {for } g \in \operatorname{supp}(\eta) W_{\epsilon} \\ 0 & \text { for } g \notin \operatorname{supp}(\eta) W_{\epsilon} .\end{cases}
$$

Proposition 5.5. For any $\ell \in \mathbb{Z}_{\geq 0}$, we have for all $0<\epsilon \ll 1$ and $y>0$,

$$
\begin{aligned}
& \phi_{\ell}^{N}\left(a_{y}\right)= \\
& \left\langle a_{y} . \phi_{\ell}, \rho_{\eta, \epsilon}\right\rangle+O\left(\epsilon(\ell+1)^{\frac{n-2}{2}} y^{n-1-\delta}+(\ell+1)^{\frac{3 n-4}{2}} y^{n-\delta}\right)+O_{\eta}\left((\ell+1)^{\frac{n-2}{2}} y^{\delta}\right)
\end{aligned}
$$

with the implied constants independent of $\ell$.

Proof. Let $h=a_{y_{0}} n_{x}^{-} m \in W_{\epsilon}$. Then for $n \in N$ and $y>0$, we have

$$
n h a_{y}=n a_{y y_{0}} n_{y x}^{-} m \text {. }
$$

As the product map $A \times N \times K \rightarrow G$ is a diffeomorphism and hence a bi-Lipschitz map in a neighborhood of $e$, there exists $q \geq 1$ such that the $\epsilon$-neighborhood of $e$ in $G$ is contained in the product $A_{q \epsilon} N_{q \epsilon} K_{q \epsilon}$ for all small $\epsilon>0$. Therefore we may write

$$
n_{y x}^{-}=a_{y_{1}} n_{x_{1}} k_{1} \in A_{q y \epsilon_{0}} N_{q y \epsilon_{0}} K_{q y \epsilon_{0}}
$$

and hence

$$
\begin{aligned}
n h a_{y}=n a_{y y_{0} y_{1}} n_{x_{1}} k_{1} m \\
\quad=n\left(a_{y y_{0} y_{1}} n_{x_{1}} a_{y y_{0} y_{1}}^{-1}\right) a_{y y_{0} y_{1}} k_{1} m=n n_{x_{1} y y_{0} y_{1}} a_{y y_{0} y_{1}} k_{1} m .
\end{aligned}
$$

By Lemma 5.3 ,

$$
\phi_{\ell}\left(n_{x} h a_{y}\right)=\phi_{\ell}\left(n_{x} n_{x_{1} y y_{0} y_{1}} a_{y y_{0} y_{1}}\right)\left(1+O\left((\ell+1)^{n-1} y\right)\right) .
$$

By Theorem 4.12, we may write $\phi_{\ell}^{N}\left(a_{y}\right)=c_{n}(\ell) y^{n-1-\delta}$ with $c_{n}(\ell) \ll$ $(\ell+1)^{(n-2) / 2}$. Hence, using Lemma 5.1 .

$$
\begin{aligned}
& \int_{(N \cap \Gamma) \backslash N} \phi_{\ell}\left(n_{x} h a_{y}\right) \cdot \eta\left(n_{x}\right) d x \\
& =\left(1+O\left((\ell+1)^{n-1} y\right)\right) \int_{N \cap \Gamma \backslash N} \phi_{\ell}\left(n_{x} n_{x_{1} y y_{0} y_{1}} a_{y y_{0} y_{1}}\right) \cdot \eta\left(n_{x}\right) d x .
\end{aligned}
$$


Since $x_{1} y y_{0} y_{1}=1+O\left(y^{2}\right)$,

$$
\begin{aligned}
& \int_{(N \cap \Gamma) \backslash N} \phi_{\ell}\left(n_{x} h a_{y}\right) \cdot \eta\left(n_{x}\right) d x \\
& =\left(1+O\left((\ell+1)^{n-1} y\right)\right) \int_{N \cap \Gamma \backslash N} \phi_{\ell}\left(n_{x} a_{y y_{0} y_{1}}\right) \cdot\left(\eta\left(n_{x}\right)+O\left(y^{2}\right)\right) d x \\
& =\int_{N \cap \Gamma \backslash N} \phi_{\ell}\left(n_{x} a_{y y_{0} y_{1}}\right) \cdot \eta\left(n_{x}\right) d x+O\left((\ell+1)^{\frac{3 n-4}{2}} y^{n-\delta}\right) \\
& \left.+O_{\eta}\left(y^{2}+(\ell+1)^{n-1} y^{3}\right)(\ell+1)^{\frac{n-2}{2}} y^{n-1-\delta}\right) \\
& =c_{n}(\ell) y^{n-1-\delta}(1+O(\epsilon))+O\left((\ell+1)^{\frac{3 n-4}{2}} y^{n-\delta}\right)+O_{\eta}\left((\ell+1)^{\frac{n-2}{2}}\left(y^{n+1-\delta}+y^{\delta}\right)\right) \\
& =c_{n}(\ell) y^{n-1-\delta}+O\left(\epsilon(\ell+1)^{\frac{n-2}{2}} y^{n-1-\delta}+(\ell+1)^{\frac{3 n-4}{2}} y^{n-\delta}\right)+O_{\eta}\left((\ell+1)^{\frac{n-2}{2}} y^{\delta}\right)
\end{aligned}
$$

as $\left|y_{0}-1\right|=O(\epsilon)$ and $\left|y_{1}-1\right|=O(y \epsilon)$.

As $\int r_{\epsilon} d \nu(h)=1$, we deduce from Lemma 5.1 that

$$
\begin{aligned}
& \left\langle a_{y} \phi_{\ell}, \rho_{\eta, \epsilon}\right\rangle=\int_{W_{\epsilon}} r_{\epsilon}(h)\left(\int_{N \cap \Gamma \backslash N} \phi_{\ell}\left(n_{x} h a_{y}\right) \eta\left(n_{x}\right) d x\right) d \nu(h) \\
& =c_{n}(\ell) y^{n-1-\delta}+O\left(\epsilon(\ell+1)^{\frac{n-2}{2}} y^{n-1-\delta}+(\ell+1)^{\frac{3 n-4}{2}} y^{n-\delta}\right)+O_{\eta}\left((\ell+1)^{\frac{n-2}{2}} y^{\delta}\right) .
\end{aligned}
$$

Since $\phi_{\ell}^{N}\left(a_{y}\right)=c_{n}(\ell) y^{n-1-\delta}$, this proves the claim.

The above proposition implies:

Corollary 5.6. For any $\ell \geq 0$,

$$
\left|\left\langle a_{y} . \phi_{\ell}, \rho_{\eta, \epsilon}\right\rangle\right| \ll(\ell+1)^{\frac{3 n-4}{2}} y^{n-1-\delta}
$$

where the implied constant is independent of $\ell \geq 0,0<\epsilon<1$ and $0<y<1$.

\section{Equidistribution of A Closed horosphere}

As before, let $n=2$ or 3 , and $G=\mathrm{PSL}_{2}(\mathbb{R}), \mathrm{PSL}_{2}(\mathbb{C})$ accordingly. Let $\Gamma<G$ be a torsion-free geometrically finite discrete subgroup with $(n-1) / 2<\delta<(n-1)$. We assume that $(N \cap \Gamma) \backslash N$ is closed. In this section, we prove the main effective equidistribution theorem for $(N \cap \Gamma) \backslash N a_{y}$ as $y \rightarrow 0$.

Let $\left\{X_{i}\right\}$ be a basis of the Lie algebra of $G$. For $\psi \in C^{\infty}(\Gamma \backslash G) \cap$ $L^{2}(\Gamma \backslash G)^{M}$, we consider the following $L^{2}$-Sobolev norm $\mathcal{S}_{m}(\psi)$ :

$$
\mathcal{S}_{m}(\psi)=\sum\|X(\psi)\|_{2}
$$


where the sum is taken over all monomials $X$ in $X_{i}$ 's of order at most $m$. By Theorem 3.1, we can fix $(n-1) / 2<s_{1}<\delta$ so that there is no eigenvalue of $\Delta$ between $s_{1}\left(n-1-s_{1}\right)$ and $\delta(n-1-\delta)$ in $L^{2}\left(\Gamma \backslash \mathbb{H}^{n}\right)$. That is, no complementary series representation of $G$ with parameter $s_{1}<s<\delta$ is contained in $L^{2}(\Gamma \backslash G)$.

Lemma 6.1. For any $\psi_{1}, \psi_{2} \in L^{2}(\Gamma \backslash G)^{M} \cap C^{\infty}(\Gamma \backslash G)$ and $0<y<1$, we have

$\left\langle a_{y} \cdot \psi_{1}, \psi_{2}\right\rangle=\sum_{\ell \in \hat{K}}\left\langle\psi_{1}, \phi_{\ell}\right\rangle\left\langle a_{y} \cdot \phi_{\ell}, \psi_{2}\right\rangle+O\left(y^{n-1-s_{1}} \cdot \mathcal{S}_{n-1}\left(\psi_{1}\right) \cdot \mathcal{S}_{n-1}\left(\psi_{2}\right)\right)$.

Proof. We write $L^{2}(\Gamma \backslash G)=V \oplus V^{\perp}$ where $V=\mathcal{H}_{\delta}$ is the complementary series representation of $G$ of parameter $\delta$. By Theorem 3.1 and the choice of $s_{1}$, the orthogonal complement $V^{\perp}$ does not contain any complementary series with parameter $s>s_{1}$. Since $V^{M}=\oplus_{\ell \in \hat{K}} \mathbb{C} \phi_{\ell}$, we can write

$$
\psi_{1}=\sum_{\ell \in \hat{K}}\left\langle\psi_{1}, \phi_{\ell}\right\rangle \phi_{\ell}+\psi_{1}^{\perp}
$$

with $\psi_{1}^{\perp} \in V^{\perp}$. Hence

$$
\left\langle a_{y} \cdot \psi_{1}, \psi_{2}\right\rangle=\sum_{\ell \in \hat{K}}\left\langle\psi_{1}, \phi_{\ell}\right\rangle\left\langle a_{y} \cdot \phi_{\ell}, \psi_{2}\right\rangle+\left\langle a_{y} \cdot \psi_{1}^{\perp}, \psi_{2}\right\rangle .
$$

On the other hand, since $V^{\perp}$ does not contain any complementary series with parameter $s>s_{1}$, we have

$$
\left|\left\langle a_{y} \cdot \psi_{1}^{\perp}, \psi_{2}\right\rangle\right| \ll y^{n-1-s_{1}} \cdot \mathcal{S}_{n-1}\left(\psi_{1}\right) \cdot \mathcal{S}_{n-1}\left(\psi_{2}\right)
$$

(see [21, Prop. 3.3] and [20, Cor. 5.6]).

We refer to [20, Lem 6.5] for the next lemma:

Lemma 6.2. For $\psi \in C_{c}^{\infty}(\Gamma \backslash G)$, there exists $\widehat{\psi} \in C_{c}^{\infty}(\Gamma \backslash G)$ such that

(1) for all small $\epsilon>0, h \in U_{\epsilon}$, and $g \in \Gamma \backslash G$,

$$
|\psi(g)-\psi(g h)| \leq \epsilon \cdot \widehat{\psi}(g) .
$$

(2) for any $i \geq 1, \mathcal{S}_{i}(\widehat{\psi}) \ll \mathcal{S}_{2 n-1}(\psi)$ with the implied constant depending only on $\operatorname{supp}(\psi)$.

Lemma 6.3. For any $\psi \in C_{c}^{\infty}(\Gamma \backslash G), \ell \geq 0$, and $i \geq 1$, we have

$$
\left|\left\langle\psi, \phi_{\ell}\right\rangle\right| \ll(\ell+1)^{-2 i}\left\|\mathcal{C}_{K}^{i} \psi\right\|_{2} .
$$

In particular, for each $i \geq 1$,

$$
\sum_{\ell \in \hat{K}}(|\ell|+1)^{2 i} \cdot\left|\left\langle\psi, \phi_{\ell}\right\rangle\right| \ll\left\|\mathcal{C}_{K}^{i+1} \psi\right\|_{2} .
$$


Proof. Recall that the operator $\mathcal{C}_{K}$ acts on each $V_{\ell}$ as a scalar $\alpha_{\ell}$, where $\alpha_{\ell}=-4 \ell^{2}$ for $n=2$ and and $\alpha_{\ell}=\ell(\ell+1)$ for $n=3$. Moreover, for any smooth vectors $v, w,\left|\left\langle\mathcal{C}_{K} v, w\right\rangle\right|=\left|\left\langle v, \mathcal{C}_{K} w\right\rangle\right|$, as $\mathcal{C}_{K}$ is skew-adjoint for $n=2$ and $\mathcal{C}_{K}$ is adjoint for $n=3$. Therefore

$$
\left|\left\langle\mathcal{C}_{K}^{i} \psi, \phi_{\ell}\right\rangle\right|=\left|\left\langle\psi, \mathcal{C}_{K}^{i} \phi_{\ell}\right\rangle\right|=\left|\alpha_{\ell}^{i}\right| \cdot\left|\left\langle\psi, \phi_{\ell}\right\rangle\right| \text {. }
$$

Hence for all $i \geq 1$,

$$
\left|\left\langle\psi, \phi_{\ell}\right\rangle\right| \leq\left|\alpha_{\ell}\right|^{-i} \cdot\left\|\mathcal{C}_{K}^{i} \psi\right\|_{2}
$$

By Theorem 4.12, we may write

$$
\phi_{\ell}^{N}\left(a_{y}\right)=c_{n}(\ell) \cdot y^{n-1-\delta}
$$

where

$$
c_{n}(\ell)=(-1)^{(n-2) \ell} \cdot c_{n}(0) \cdot \frac{\sqrt{\Gamma(n-1-\delta) \Gamma(\ell+\delta)}}{\sqrt{\Gamma(\delta) \Gamma(\ell+n-1-\delta)}} \cdot \sqrt{2(n-2) \ell+1} .
$$

Theorem 6.5. Fix $0<\mathbf{s}_{\Gamma}<\delta-s_{1}$. For any $\psi \in C_{c}^{\infty}(\Gamma \backslash G)^{M}$,

$$
\psi^{N}\left(a_{y}\right)=\sum_{\ell \in \hat{K}} c_{n}(\ell)\left\langle\psi, \phi_{\ell}\right\rangle y^{n-1-\delta}+\mathcal{S}_{2 n-1}(\psi) O\left(y^{n-1-\delta+\frac{2 \mathbf{s}_{\Gamma}}{(2 n+1)}}\right) .
$$

Proof. Fix $\psi \in C_{c}^{\infty}(\Gamma \backslash G)^{M}$. When $\infty$ has rank $n-1$, we set $J=F_{0}$. In other cases, it was shown in 20] that there exists a bounded open subset $J$ of $F_{0}$ such that $\psi\left(n_{x} a_{y}\right)=0$ for all $x \in F_{0}-J$ and all $0<y<1$. We assume that $J$ contains $F_{\Lambda}$, which is a bounded fundamental domain for the action of $(N \cap \Gamma)$ in $\Lambda(\Gamma)-\{\infty\}$.

Choose a non-negative function $\eta \in C_{c}^{\infty}(N \cap \Gamma \backslash N)$ such that $\left.\eta\right|_{J}=1$. Then

$$
I_{\eta}(\psi)\left(a_{y}\right):=\int_{(N \cap \Gamma) \backslash N} \psi\left(n_{x} a_{y}\right) \eta\left(n_{x}\right) d x=\psi^{N}\left(a_{y}\right) .
$$

Let $\epsilon_{0}, W_{\epsilon}, r_{\epsilon}, \rho_{\eta, \epsilon}$ be as defined in section 5 with respect to $J$ and $\eta$. Since $r_{\epsilon}$ is the approximation of the identity in $A$ direction, $\mathcal{S}_{n-1}\left(\rho_{\eta, \epsilon}\right)=$ $O_{\eta}\left(\epsilon^{(-2 n+1) / 2}\right)$. For any $0<y<1$, and any small $\epsilon>0$, we have (see the proof of Prop. 6.6 in [20])

$$
\left|I_{\eta}(\psi)\left(a_{y}\right)-\left\langle a_{y} \cdot \psi, \rho_{\eta, \epsilon}\right\rangle\right| \ll(\epsilon+y) \cdot I_{\eta}(\widehat{\psi})\left(a_{y}\right) .
$$

Setting $\psi_{0}(g):=\psi(g)$, we define for $1 \leq i \leq k$, inductively

$$
\psi_{i}(g):=\widehat{\psi}_{i-1}(g)
$$

where $\widehat{\psi}_{i-1}$ is given by Lemma 6.2 .

Let $k$ be an integer bigger than $1+\frac{(n-1-\delta)(2 n+1)}{2\left(\delta-s_{1}\right)}$. 
Applying Lemma 6.2 to each $\psi_{i}$, we obtain for $0 \leq i \leq k-1$,

$$
\begin{aligned}
I_{\eta}\left(\psi_{i}\right)\left(a_{y}\right) & =\left\langle a_{y} \cdot \psi_{i}, \rho_{\eta, \epsilon}\right\rangle+O\left((\epsilon+y) \cdot I_{\eta}\left(\widehat{\psi}_{i}\right)\left(a_{y}\right)\right) \\
& =\left\langle a_{y} \cdot \psi_{i}, \rho_{\eta, \epsilon}\right\rangle+O\left((\epsilon+y) \cdot I_{\eta}\left(\psi_{i+1}\right)\left(a_{y}\right)\right)
\end{aligned}
$$

and

$$
I_{\eta}\left(\psi_{k}\right)\left(a_{y}\right)=\left\langle a_{y} \cdot \psi_{k}, \rho_{\eta, \epsilon}\right\rangle+O_{\eta}\left((\epsilon+y) \mathcal{S}_{n-1}\left(\psi_{k}\right)\right) .
$$

By Lemma 6.3.

$$
\left|\left\langle\psi_{i}, \phi_{\ell}\right\rangle\right|=O(|\ell|+1)^{-(2 n-2)} \mathcal{S}_{2 n-2}\left(\psi_{i}\right)
$$

and by Lemma $6.2, \mathcal{S}_{j}\left(\psi_{i}\right) \ll \mathcal{S}_{2 n-1}(\psi)$ for all $j \geq 1$.

Since $\left|\left\langle a_{y} . \phi_{\ell}, \rho_{\eta, \epsilon}\right\rangle\right| \ll(|\ell|+1)^{(3 n-4) / 2} y^{n-1-\delta}$ by Corollary 5.6. we have

$$
\sum_{\ell \in \hat{K}}\left\langle\psi_{i}, \phi_{\ell}\right\rangle\left\langle a_{y} \cdot \phi_{\ell}, \rho_{\eta, \epsilon}\right\rangle=O\left(\mathcal{S}_{2 n-1}\left(\psi_{i}\right) \cdot y^{n-1-\delta}\right)
$$

By Lemma 6.1, we deduce that for each $1 \leq i \leq k-1$,

$$
\begin{aligned}
\left\langle a_{y} \cdot \psi_{i}, \rho_{\eta, \epsilon}\right\rangle & =\sum_{\ell \in \hat{K}}\left\langle\psi_{i}, \phi_{\ell}\right\rangle\left\langle a_{y} \cdot \phi_{\ell}, \rho_{\eta, \epsilon}\right\rangle+O\left(y^{n-1-s_{1}} \cdot \mathcal{S}_{n-1}\left(\psi_{i}\right) \cdot \mathcal{S}_{n-1}\left(\rho_{\eta, \epsilon}\right)\right) \\
& =O\left(\mathcal{S}_{2 n-1}(\psi) \cdot y^{n-1-\delta}\right)+O\left(y^{n-1-s_{1}} \cdot \mathcal{S}_{n-1}\left(\psi_{i}\right) \mathcal{S}_{n-1}\left(\rho_{\eta, \epsilon}\right)\right) \\
& =\mathcal{S}_{2 n-1}(\psi) \cdot O\left(y^{n-1-\delta}+\epsilon^{-(2 n-1) / 2} y^{n-1-s_{1}}\right) .
\end{aligned}
$$

Hence for any $0<y<\epsilon$, using Proposition 5.5, we deduce

$$
\begin{aligned}
& I_{\eta}(\psi)\left(a_{y}\right)=\left\langle a_{y} \cdot \psi, \rho_{\eta, \epsilon}\right\rangle+\sum_{j=1}^{k-1} O\left(\left\langle a_{y} \cdot \psi_{j}, \rho_{\eta, \epsilon}\right\rangle(\epsilon+y)^{j}\right)+O_{\psi}\left((\epsilon+y)^{k}\right) \\
& =\left\langle a_{y} \cdot \psi, \rho_{\eta, \epsilon}\right\rangle+\mathcal{S}_{2 n-1}(\psi) O\left(\epsilon \cdot y^{n-1-\delta}+\epsilon^{-(2 n-1) / 2} y^{n-1-s_{1}}+\epsilon^{k}\right) \\
& =\sum_{\ell \in \hat{K}}\left\langle\psi, \phi_{\ell}\right\rangle\left\langle a_{y} \cdot \phi_{\ell}, \rho_{\eta, \epsilon}\right\rangle+\mathcal{S}_{2 n-1}(\psi) O\left(\epsilon \cdot y^{n-1-\delta}+\epsilon^{-(2 n-1) / 2} y^{n-1-s_{1}}+\epsilon^{k}\right) \\
& =\sum_{\ell \in \hat{K}}\left\langle\psi, \phi_{\ell}\right\rangle c_{n}(\ell) y^{n-1-\delta}+ \\
& \sum_{\ell \in \hat{K}}\left\langle\psi, \phi_{\ell}\right\rangle\left(O\left(\epsilon(|\ell|+1)^{\frac{n-2}{2}} y^{n-1-\delta}+(|\ell|+1)^{\frac{3 n-4}{2}} y^{n-\delta}\right)+O_{\eta}\left((|\ell|+1)^{(n-2) / 2} y^{\delta}\right)\right) \\
& \quad+\mathcal{S}_{2 n-1}(\psi) O\left(y^{\delta}+\epsilon y^{n-1-\delta}+\epsilon^{-(2 n-1) / 2} y^{n-1-s_{1}}+\epsilon^{k}\right) .
\end{aligned}
$$

Since $\left|\left\langle\psi, \phi_{\ell}\right\rangle\right| \ll(|\ell|+1)^{-(2 n-2)} O\left(\mathcal{S}_{2 n-2}(\psi)\right)$, we have

$$
\sum_{\ell \in \hat{K}}\left\langle\psi, \phi_{\ell}\right\rangle(|\ell|+1)^{(3 n-4) / 2}=O(1), \quad \sum_{\ell \in \hat{K}}\left\langle\psi, \phi_{\ell}\right\rangle(|\ell|+1)^{(n-2) / 2}=O(1) .
$$


Hence we deduce

$$
\begin{aligned}
& I_{\eta}(\psi)\left(a_{y}\right)=\sum_{\ell \in \hat{K}}\left\langle\psi, \phi_{\ell}\right\rangle c_{n}(\ell) y^{n-1-\delta} \\
& \quad+\mathcal{S}_{2 n-1}(\psi) O\left(\epsilon y^{n-1-\delta}+y^{n-\delta}+y^{\delta}+\epsilon^{-(2 n-1) / 2} y^{n-1-s_{1}}+\epsilon^{k}\right) .
\end{aligned}
$$

By equating $\epsilon \cdot y^{n-1-\delta}$ and $\epsilon^{-(2 n-1) / 2} y^{n-1-s_{1}}$, we put $\epsilon=y^{2\left(\delta-s_{1}\right) /(2 n+1)}$ and obtain

$$
I_{\eta}(\psi)\left(a_{y}\right)=\sum_{\ell \in \hat{K}}\left\langle\psi, \phi_{\ell}\right\rangle c_{n}(\ell) y^{n-1-\delta}+\mathcal{S}_{2 n-1}(\psi) O\left(y^{n-1-\delta+\frac{2\left(\delta-s_{1}\right)}{2 n+1}}\right) .
$$

\section{COMPARING MAIN TERMS FROM DIFFERENT APPROACHES}

The coefficient $\sum_{\ell \in \hat{K}} c_{n}(\ell)\left\langle\psi, \phi_{\ell}\right\rangle$ of the main term in Theorem 6.5 is related to the space average of $\psi$ with respect to the Burger-Roblin measure (which we will call the BR measure for short) by 30] and [20] (also see [26]).

Recall that $\phi_{0}=\phi_{0}^{\Gamma}$ is given by

$$
\phi_{0}(x+j y)=\int_{\mathbb{R}^{n-1}}\left(\frac{\left(|u|^{2}+1\right) y}{|x-u|^{2}+y^{2}}\right)^{\delta} d \nu_{j}(u)
$$

for the Patterson-Sullivan measure $\nu_{j}=\nu_{j}^{\Gamma}$ on the boundary $\partial\left(\mathbb{H}^{n}\right)$. Note that $\phi_{0}^{\Gamma}(j)=\left|\nu_{j}^{\Gamma}\right|$. As before, we normalize $\nu_{j}$ so that $\left\|\phi_{0}\right\|_{2}=1$.

For $\xi \in \partial\left(\mathbb{H}^{n}\right)$ and $z_{1}, z_{2} \in \mathbb{H}^{n}$, recall the Busemann function:

$$
\beta_{\xi}\left(z_{1}, z_{2}\right)=\lim _{s \rightarrow \infty} d\left(z_{1}, \xi_{s}\right)-d\left(z_{2}, \xi_{s}\right)
$$

where $\xi_{s}$ is a geodesic ray tending to $\xi$ as $s \rightarrow \infty$. Using the identification of $\mathrm{T}^{1}\left(\mathbb{H}^{n}\right)$ and $G / M$, we give the definition of the BowenMargulis-Sullivan measure $m^{\mathrm{BMS}}$ on $\Gamma \backslash G / M$. For $u \in \mathrm{T}^{1}\left(\mathbb{H}^{n}\right)$, we denote by $u^{+}$and $u^{-}$the forward and the backward endpoints of the geodesic determined by $u$, respectively. The correspondence $u \mapsto$ $\left(u^{+}, u^{-}, \beta_{u^{-}}(j, \pi(u))\right)$ gives a homeomorphism between the space $\mathrm{T}^{1}\left(\mathbb{H}^{n}\right)$ with $\left(\partial\left(\mathbb{H}^{n}\right) \times \partial\left(\mathbb{H}^{n}\right)-\left\{(\xi, \xi): \xi \in \partial\left(\mathbb{H}^{n}\right)\right\}\right) \times \mathbb{R}$ where $\pi: G \rightarrow G / K=$ $\mathbb{H}^{n}$ is the canonical projection. Define the measure $\tilde{m}^{\mathrm{BMS}}$ on $G / M$ :

$$
d \tilde{m}^{\mathrm{BMS}}(u)=e^{\delta \beta_{u^{+}}(j, \pi(u))} e^{\delta \beta_{u^{-}}(j, \pi(u))} d \nu_{j}\left(u^{+}\right) d \nu_{j}\left(u^{-}\right) d t
$$

where $t=\beta_{u^{-}}(j, \pi(u))$. This measure is left $\Gamma$-invariant and hence induces a measure $m^{\mathrm{BMS}}$ on $\Gamma \backslash G / M$.

Roblin obtained the following interesting identity in his thesis [31]: 
Theorem 7.1 (Roblin). For $\delta>(n-1) / 2$,

$$
\left\|\phi_{0}\right\|_{2}^{2}=\left|m^{\mathrm{BMS}}\right| \cdot \int_{\mathbb{R}^{n-1}} \frac{d x}{\left(1+|x|^{2}\right)^{\delta}}
$$

As we have normalized $\nu_{j}$ so that $\left\|\phi_{0}\right\|_{2}=1$ and $\phi_{0}(j)=\left|\nu_{j}\right|$, we deduce

$$
\frac{1}{\mid m^{\mathrm{BMS} \mid}}=\int_{\mathbb{R}^{n-1}} \frac{d x}{\left(1+|x|^{2}\right)^{\delta}} .
$$

To describe the equidistribution result of $(N \cap \Gamma) \backslash N a_{y}$ from [30], we recall the measure $\tilde{m}_{N}^{\mathrm{BR}}$ defined in the introduction: for $\psi \in C_{c}(G / M)$,

$$
\tilde{m}_{N}^{\mathrm{BR}}(\psi)=\int_{K A N} \psi\left(k a_{y} n_{x}\right) y^{\delta-1} d x d y d \nu_{j}(k(0)) .
$$

The BR measure $m_{N}^{\mathrm{BR}}$ (associated to the stable horospherical subgroup $N)$ is the measure on $\Gamma \backslash G / M$ induced from $\tilde{m}_{N}^{\mathrm{BR}}$.

We define the measure $\mu_{N}^{\mathrm{PS}}$ on $N$ by

$$
d \mu_{N}^{\mathrm{PS}}\left(n_{x}\right)=e^{-\delta \beta_{x}(j, x+j)} d \nu_{j}(x)=\left(1+|x|^{2}\right)^{\delta} d \nu_{j}(x) .
$$

This induces a measure on $(N \cap \Gamma) \backslash N$ for which we use the same notation $\mu_{N}^{\mathrm{PS}}$. Since $\mu_{N}^{\mathrm{PS}}$ is supported in $(N \cap \Gamma) \backslash(\Lambda(\Gamma)-\{\infty\})$, which is compact, we have $\mu_{N}^{\mathrm{PS}}((N \cap \Gamma) \backslash N)<\infty$.

The following is proved by Roblin [30] when $(N \cap \Gamma) \backslash N$ is compact and in [26] in general.

Theorem 7.2. Let $\delta>0$ and $(N \cap \Gamma) \backslash N$ closed. For any $\psi \in$ $C_{c}(\Gamma \backslash G)^{M}$,

$$
\lim _{y \rightarrow 0} y^{\delta-n+1} \cdot \psi^{N}\left(a_{y}\right)=\frac{\mu_{N}^{\mathrm{PS}}(N \cap \Gamma \backslash N)}{\left|m^{\mathrm{BMS}}\right|} m_{N}^{\mathrm{BR}}(\psi) .
$$

Comparing the coefficients of the main terms of Theorem 7.2 and Theorem 6.5, and using Theorem 7.1, we deduce the following identity of the Burger-Roblin measure considered as a distribution on $\Gamma \backslash G$ :

Theorem 7.3. Let $\delta>(n-1) / 2$. For any $\psi \in C_{c}^{\infty}(\Gamma \backslash G)$,

$$
\kappa_{\Gamma} \cdot m_{N}^{\mathrm{BR}}(\psi)=\sum_{\ell \in \hat{K}} c_{n}(\ell)\left\langle\psi, \phi_{\ell}\right\rangle
$$

with $c_{n}(\ell)$ as in $(6.4)$ and $\kappa_{\Gamma}=\int_{\mathbb{R}^{n-1}} \frac{d x}{\left(1+|x|^{2}\right)^{\delta}} \cdot \int_{n_{x} \in(N \cap \Gamma) \backslash N}\left(1+|x|^{2}\right)^{\delta} d \nu_{j}(x)$.

Now Theorem 1.6 is a direct consequence of Theorem 7.3 and Theorem 1.3 . 


\section{Application to COUnting in SECtors}

Let $n=2$ or 3 . Let $Q$ be a real quadratic form of signature $(n, 1)$ and $v_{0} \in \mathbb{R}^{n+1}$ be a non-zero vector such that $Q\left(v_{0}\right)=0$. Let $\Gamma_{0}$ be a geometrically finite subgroup of the identity component of $\mathrm{SO}_{Q}(\mathbb{R})$. Suppose that $\delta>(n-1) / 2$ and that $v_{0} \Gamma_{0}$ is discrete.

Let $\|\cdot\|$ be any norm in $\mathbb{R}^{n+1}$ and set $B_{T}:=\left\{v \in \mathbb{R}^{n+1}:\|v\|<T\right\}$. Let $G=\mathrm{PSL}_{2}(\mathbb{R})$ if $n=2$ and $\mathrm{PSL}_{2}(\mathbb{C})$ if $n=3$. Let $\iota: G \rightarrow \mathrm{SO}_{Q}(\mathbb{R})$ be a representation so that the stabilizer of $v_{0}$ in $G$ via $\iota$ is $N M$. Let $\Gamma:=\iota^{-1}\left(\Gamma_{0}\right)$.

8.1. Counting I. For $g \in G$, we write $\kappa(g)$ for the $K$-coordinate of $g$ in the Iwasawa decomposition $G=N A K$. As before, $M$ denotes the centralizer of $A$ in $K$. Fixing a function $f$ on $M \backslash K$, define the counting function $F_{T}$ on $\Gamma \backslash G$ by

$$
F_{T}(g)=\sum_{\gamma \in N \cap \Gamma \backslash \Gamma} \chi_{B_{T}}\left(v_{0} \gamma g\right) f([\kappa(\gamma g)])
$$

where $\chi_{B_{T}}$ denotes the characteristic function of $B_{T}$. Since $\kappa(g)=$ $\kappa(n g)$ for any $n \in N$ and $g \in G, F_{T}$ is well-defined.

For $k \in K$ and $\psi \in C_{c}(G)$, define $\psi^{k} \in C_{c}(G)^{M}$ by

$$
\psi^{k}(g)=\int_{m \in M} \psi(g m k) d m
$$

where $d m$ denotes the probability Haar measure of $M$. Similarly, for $\Psi \in C_{c}(\Gamma \backslash G)$, we set $\Psi^{k}(g)=\int_{m \in M} \Psi(g m k) d m$.

Lemma 8.1. For $\Psi \in C_{c}(\Gamma \backslash G)$ and for any Borel function $f$ on $K$, we have

$$
\left\langle F_{T}, \Psi\right\rangle=\int_{k \in M \backslash K} f([k]) \int_{y>\left\|v_{0} k\right\| T^{-1}}\left(\int_{(N \cap \Gamma) \backslash N} \Psi^{k}\left(n_{x} a_{y}\right) d x\right) y^{-n} d y d k
$$

where $d k$ denotes the probability Haar measure on $K$, also understood as the invariant measure on $M \backslash K$. 
Proof. We compute

$$
\begin{aligned}
& \left\langle F_{T}, \Psi\right\rangle=\int_{\Gamma \backslash G} \sum_{\gamma \in N \cap \Gamma \backslash \Gamma} \chi_{B_{T}}\left(v_{0} \gamma g\right) f(\kappa(\gamma g)) \Psi(g) d g \\
& =\int_{(N \cap \Gamma) \backslash G} \chi_{B_{T}}\left(v_{0} g\right) f(\kappa(g)) \Psi(g) d g \\
& =\int_{a_{y} k \in A K} \chi_{B_{T}}\left(v_{0} a_{y} k\right) f(k)\left(\int_{(N \cap \Gamma) \backslash N} \Psi\left(n_{x} a_{y} k\right) d x\right) y^{-n} d y d k \\
& =\int_{k \in M \backslash K} \int_{y>\left\|v_{0} k\right\| T^{-1}} f(k)\left(\int_{(N \cap \Gamma) \backslash N}\left(\int_{m \in M} \Psi\left(n_{x} a_{y} m k\right) d m\right) d x\right) y^{-n} d y d k .
\end{aligned}
$$

By Theorem 1.6, for any $\Psi \in C^{\infty}(\Gamma \backslash G)^{M}$ and $k \in M \backslash K$, we have, as $y \rightarrow 0$,

$$
\int_{(N \cap \Gamma) \backslash N} \Psi^{k}\left(n_{x} a_{y}\right) d x=\kappa_{\Gamma} \cdot m_{N}^{\mathrm{BR}}\left(\Psi^{k}\right) \cdot y^{n-1-\delta}+\mathcal{S}_{2 n-1}(\Psi) O\left(y^{(n-1-\delta)+\frac{2 \mathbf{s}_{\Gamma}}{2 n+1}}\right) .
$$

Therefore, we deduce from Lemma 8.1.

Theorem 8.2. For any $\Psi \in C^{\infty}(\Gamma \backslash G)$ and a bounded Borel function $f$ on $M \backslash K$, we have, as $T \rightarrow \infty$,

$$
\left\langle F_{T}, \Psi\right\rangle=\frac{\kappa_{\Gamma}}{\delta} \cdot\left(\int_{k \in M \backslash K} \frac{m_{N}^{\mathrm{BR}}\left(\Psi^{k}\right) \cdot f(k)}{\left\|v_{0} k\right\|^{\delta}} d k\right) \cdot T^{\delta}+O\left(\mathcal{S}_{2 n-1}(\Psi) T^{\delta-\frac{2 \mathbf{s}_{\Gamma}}{2 n+1}}\right) .
$$

8.2. Counting II. For a left $M$-invariant Borel subset $\Omega \subset K$ and $T>0$, define

$$
S_{T}(\Omega):=\left\{v \in v_{0} A \Omega:\|v\|<T\right\} .
$$

For a subset $I$ of $\mathbb{Z}_{\geq 0}$, let $\left\{\Gamma_{d}<\Gamma_{0}: d \in I\right\}$ be a family of subgroups of finite index which satisfies $\operatorname{Stab}_{\Gamma_{0}} v_{0}=\operatorname{Stab}_{\Gamma_{d}} v_{0}$ and which has a uniform spectral gap, say, $\mathbf{s}_{0}$.

Set

$$
\Xi_{v_{0}}\left(\Gamma_{0}, \Omega\right):=\frac{\kappa_{\iota^{-1}\left(\Gamma_{0}\right)}}{\delta} \int_{k^{-1} \in \Omega} \frac{d \nu_{j}^{\Gamma}(k(0))}{\left\|v_{0} k^{-1}\right\|^{\delta}} .
$$

We deduce the following from Theorem 8.2 .

Theorem 8.4. Let $\Omega$ be an admissible $M$-invariant Borel subset of $K$ and let $q_{\Omega}>0$ be as in (1.7). For any $\gamma^{\prime} \in \Gamma_{0}$,

$$
\#\left(v_{0} \Gamma_{d} \gamma^{\prime} \cap S_{T}(\Omega)\right)=\frac{\Xi_{v_{0}}\left(\Gamma_{0}, \Omega\right)}{\left[\Gamma_{0} \Gamma_{d}\right]} T^{\delta}+O\left(T^{\delta-\frac{8 \mathbf{s}_{0}}{n(n+9)(2 n+1) q_{\Omega}}}\right)
$$

with the implied constant independent of $d$ and $\gamma^{\prime}$. 
Proof. Let $\Gamma:=\iota^{-1}\left(\Gamma_{0}\right)$ and $\gamma_{0}:=\iota^{-1}\left(\gamma^{\prime}\right)$. Recall that $U_{\epsilon}$ denotes an $\epsilon$-neighborhood of $e$ in $G$. By abuse of notation, we use the notation $\Gamma_{d}$ to denote $\iota^{-1}\left(\Gamma_{d}\right)$. Moreover, for all sufficiently small $\epsilon>0$, and for $K_{\epsilon}:=U_{\epsilon} \cap K$, we have, for $\Omega_{\epsilon+}=\Omega K_{\epsilon}$ and $\Omega_{\epsilon-}=\cap_{k \in K_{\epsilon}} \Omega k$,

$$
\nu_{j}\left(\Omega_{\epsilon+}^{-1}(0)-\Omega_{\epsilon-}^{-1}(0)\right)=O\left(\epsilon^{q_{\Omega}^{\prime}}\right) .
$$

By the strong wave front lemma [14, Theorem 4.1], there exists $0<$ $\ell_{0}<1$ such that for $T \gg 1$,

$$
S_{T}(\Omega) U_{\ell_{0} \epsilon} \subset S_{(1+\epsilon) T}\left(\Omega_{\epsilon+}\right) \quad \text { and } \quad S_{(1-\epsilon) T}\left(\Omega_{\epsilon-}\right) \subset \cap_{u \in U_{\ell_{0}}} S_{T}(\Omega) u .
$$

Let $\psi_{\epsilon} \in C_{c}^{\infty}(G)$ be a non-negative function supported in $U_{\ell_{0} \epsilon}$ with integral one, and define the following function of $\Gamma_{d} \backslash G$ :

$$
\Psi_{\Gamma_{d}, \epsilon}(g)=\sum_{\gamma \in \Gamma_{d}} \psi_{\epsilon}(\gamma g)
$$

Define the counting function $F_{T}^{\Omega}$ on $\Gamma_{d} \backslash G$ by

$$
F_{T}^{\Omega}(g)=\sum_{\gamma \in N \cap \Gamma \backslash \Gamma_{d}} \chi_{S_{T}(\Omega)}\left(v_{0} \gamma g\right)=\sum_{\gamma \in N \cap \Gamma \backslash \Gamma_{d}} \chi_{B_{T}}\left(v_{0} \gamma g\right) \chi_{\Omega}([\kappa(\gamma g)]) ;
$$

this is well-defined as $\Gamma_{d} \cap N=\Gamma \cap N$ by the assumption on $\Gamma_{d}$. Note that $F_{T}^{\Omega}\left(\gamma_{0}\right)=\# v_{0} \Gamma_{d} \gamma_{0} \cap S_{T}(\Omega)$ and that

$$
F_{(1-\epsilon) T}^{\Omega_{\epsilon-}}\left(\gamma_{0} g\right) \leq F_{T}^{\Omega}\left(\gamma_{0}\right) \leq F_{(1+\epsilon) T}^{\Omega_{\epsilon+}}\left(\gamma_{0} g\right)
$$

for all $g \in U_{\ell_{0} \epsilon}$. On the other hand,

$$
\int_{\Gamma_{d} \backslash G} F_{(1 \pm \epsilon) T}^{\Omega_{\epsilon \pm}}\left(\gamma_{0} g\right) \Psi_{\Gamma_{d}, \epsilon}(g) d g=\int_{\Gamma_{d} \backslash G} F_{(1 \pm \epsilon) T}^{\Omega_{\epsilon \pm}}(g) \Psi_{\Gamma_{d}, \epsilon}\left(\gamma_{0}^{-1} g\right) d g .
$$

Therefore, if we set $\Psi_{\Gamma_{d}, \epsilon}^{\gamma_{0}}(g):=\Psi_{\Gamma_{d}, \epsilon}\left(\gamma_{0}^{-1} g\right)$, we have

$$
\left\langle F_{(1-\epsilon) T}^{\Omega_{\epsilon-}}, \Psi_{\Gamma_{d}, \epsilon}^{\gamma_{0}}\right\rangle \leq F_{T}^{\Omega}\left(\gamma_{0}\right) \leq\left\langle F_{(1+\epsilon) T}^{\Omega_{\epsilon+}}, \Psi_{\Gamma_{d}, \epsilon}^{\gamma_{0}}\right\rangle
$$

where the inner product has taken place in $L^{2}\left(\Gamma_{d} \backslash G\right)$. Since $\phi_{0}^{\Gamma}(e)=$ $\left|\nu_{j}^{\Gamma}\right|$ and $\left\|\phi_{0}^{\Gamma}\right\|_{2}=1$, we note that for all positive integer $d$,

$$
\nu_{j}^{\Gamma_{d}}=\frac{1}{\sqrt{\left[\Gamma: \Gamma_{d}\right]}} \nu_{j}^{\Gamma} .
$$

Therefore it follows that $\kappa_{\iota}{ }^{-1}\left(\Gamma_{d}\right)=\frac{1}{\sqrt{\left[\Gamma: \Gamma_{d}\right]}} \kappa_{\iota^{-1}}(\Gamma)$, and that for any $\Gamma$-invariant continuous function $f$ on $G / M$,

$$
m_{\Gamma_{d}, N}^{\mathrm{BR}}(f)=\frac{1}{\sqrt{\left[\Gamma: \Gamma_{d}\right]}} m_{\Gamma, N}^{\mathrm{BR}}(f) .
$$

We use the following (see [26, Prop. 6.2], or [20, Sec. 7]):

$$
\int_{k \in \Omega} \frac{m_{\Gamma, N}^{\mathrm{BR}}\left(\Psi_{\Gamma, \epsilon}^{k}\right)}{\left\|v_{0} k\right\|^{\delta}} d k=\int_{k \in \Omega^{-1}} \frac{d \nu_{j}^{\Gamma}(k(0))}{\left\|v_{0} k^{-1}\right\|^{\delta}} \cdot(1+O(\epsilon)) .
$$


Since $\operatorname{dim}(G)=n(n+1) / 2$, we compute $\mathcal{S}_{2 n-1}\left(\Psi_{\epsilon}\right)=O\left(\epsilon^{-\left(n^{2}+9 n-4\right) / 4}\right)$. Hence putting these together and using Theorem 8.2, we have

$$
\begin{aligned}
& \left\langle F_{(1 \pm \epsilon) T}^{\Omega_{\epsilon \pm}}, \Psi_{\Gamma_{d}, \epsilon}^{\gamma_{0}}\right\rangle \\
& =\frac{\kappa_{\Gamma} \cdot(1 \pm \epsilon)^{\delta}}{\delta\left[\Gamma: \Gamma_{d}\right]} T^{\delta} \int_{k \in \Omega_{\epsilon \pm}^{-1}} \frac{d \nu_{j}(k(0))}{\left\|v_{0} k^{-1}\right\|^{\delta}}+O\left(\epsilon T^{\delta}+\epsilon^{-\left(n^{2}+9 n-4\right) / 4} T^{\delta-\frac{2 \mathbf{s}_{0}}{2 n+1}}\right) \\
& =\frac{\kappa_{\Gamma} \cdot T^{\delta}}{\delta\left[\Gamma: \Gamma_{d}\right]} \int_{k \in \Omega_{\epsilon \pm}^{-1}} \frac{d \nu_{j}(k(0))}{\left\|v_{0} k^{-1}\right\| \delta}+O\left(\epsilon T^{\delta}+\epsilon^{-\left(n^{2}+9 n-4\right) / 4} T^{\delta-\frac{2 \mathbf{s}_{0}}{2 n+1}}\right) \\
& =\frac{\kappa_{\Gamma} \cdot T^{\delta}}{\delta\left[\Gamma: \Gamma_{d}\right]} \int_{k \in \Omega^{-1}} \frac{d \nu_{j}(k(0))}{\left\|v_{0} k^{-1}\right\|^{\delta}}+O\left(\epsilon^{q_{\Omega}} T^{\delta}+\epsilon^{-\left(n^{2}+9 n-4\right) / 4} T^{\delta-\frac{2 \mathbf{s}_{0}}{2 n+1}}\right) .
\end{aligned}
$$

Hence, by equating $\epsilon^{-\left(n^{2}+9 n-4\right) / 4} T^{-\frac{2 \mathbf{s}_{0}}{(2 n+1)}}=\epsilon^{q_{\Omega}}$, we deduce

$$
F_{T}^{\Omega}(e)=\frac{\kappa_{\Gamma} \cdot T^{\delta}}{\delta\left[\Gamma: \Gamma_{d}\right]} \int_{k \in \Omega^{-1}} \frac{d \nu_{j}(k(0))}{\left\|v_{0} k^{-1}\right\|^{\delta}}+O\left(T^{\delta-\frac{8 \mathbf{s}_{0}}{n(n+9)(2 n+1) q_{\Omega}}}\right) .
$$

This finishes the proof of Theorem 8.4 .

8.3. Let $\mathcal{P}$ be an Apollonian packing as in Theorem 1.1. Let

$$
Q\left(x_{1}, x_{2}, x_{3}, x_{4}\right)=2\left(x_{1}^{2}+x_{2}^{2}+x_{3}^{2}+x_{4}^{2}\right)-\left(x_{1}+x_{2}+x_{3}+x_{4}\right)^{2}
$$

be the Descartes quadratic form, which has signature $(3,1)$. Let $\mathcal{A}$ denote the Apollonian group, i.e., the subgroup of $O_{Q}(\mathbb{Z})$ generated by

$$
\begin{aligned}
& S_{1}=\left(\begin{array}{cccc}
-1 & 0 & 0 & 0 \\
2 & 1 & 0 & 0 \\
2 & 0 & 1 & 0 \\
2 & 0 & 0 & 1
\end{array}\right), S_{2}=\left(\begin{array}{cccc}
1 & 2 & 0 & 0 \\
0 & -1 & 0 & 0 \\
0 & 2 & 1 & 0 \\
0 & 2 & 0 & 1
\end{array}\right), \\
& S_{3}=\left(\begin{array}{cccc}
1 & 0 & 2 & 0 \\
0 & 1 & 2 & 0 \\
0 & 0 & -1 & 0 \\
0 & 0 & 2 & 1
\end{array}\right), \quad S_{4}=\left(\begin{array}{cccc}
1 & 0 & 0 & 2 \\
0 & 1 & 0 & 2 \\
0 & 0 & 1 & 2 \\
0 & 0 & 0 & -1
\end{array}\right) .
\end{aligned}
$$

The critical exponent of $\mathcal{A}$ is equal to $\alpha$, which is the Hausdorff dimension of the residual set of $\mathcal{P}$ and is a geometrically finite group (cf. [20]).

In [20, Sec. 2], it was shown that there exists a vector $v_{0}$ with $Q\left(v_{0}\right)=0$, whose coordinates are given by the curvatures of four mutually tangent circles of $\mathcal{P}$ and that

$$
N_{T}(\mathcal{P})=\left\{v \in v_{0} \mathcal{A}:\|v\|_{\max }<T\right\}+3
$$

for all $T \gg 1$.

Therefore Theorem 8.4 implies, as $q_{K}=1$ : 
Corollary 8.7. For some $c_{\mathcal{P}}>0$,

$$
N_{T}(\mathcal{P})=c_{\mathcal{P}} \cdot T^{\alpha}+O\left(T^{\alpha-2 \mathbf{s}_{\mathcal{A}} / 63}\right)
$$

Moreover, if we set $\mathcal{A}_{0}<\mathrm{SO}(Q)^{\circ}$ to be a torsion free finite index subgroup of $\mathcal{A}$ and write $v_{0} \mathcal{A}$ as the disjoint union $\cup_{i=1}^{m} v_{i} \mathcal{A}_{0}$, then

$$
c_{\mathcal{P}}=\sum_{i=1}^{m} \Xi_{v_{i}}\left(\mathcal{A}_{0}, K\right)
$$

where $\Xi_{v_{i}}$ is defined as in 8.3 .

On the other hand, it can be deduced from the main results in [27] that

$$
\lim _{T \rightarrow \infty} \frac{N_{T}(\mathcal{P})}{T^{\alpha}}=c_{A} \cdot \mathcal{H}_{\alpha}(\operatorname{Res}(\mathcal{P}))
$$

where $c_{A}>0$ is a constant independent of $\mathcal{P}$ (cf. [28] for details). Therefore Theorem 1.1 follows from Corollary 8.7 .

\section{REFERENCES}

[1] Daniel Bump. Automorphic forms and Representations. Camb. Studies in Advanced Matg., 55, 1998

[2] Marc Burger. Horocycle flow on geometrically finite surfaces. Duke Math. J., 61(3):779-803, 1990.

[3] Jean Bourgain and Elena Fuchs. A proof of the positive density conjecture for integer Apollonian packings J. Amer. Math. Soc. 24, 945-967, 2011

[4] Jean Bourgain and Alex Kontorovich. On the strong density conjecture of Apollonian packings Preprint. arXive:1205:4416, 2012

[5] Jean Bourgain, Alex Gamburd, and Peter Sarnak. Generalization of Selberg's $3 / 16$ theorem and Affine sieve To appear in Acta $M$.

[6] Jean Bourgain, Alex Gamburd, and Peter Sarnak. Affine linear sieve, expanders, and sum-product Inventiones 179, (2010) 559-644

[7] Jean Bourgain, Alex Kontorovich, and Peter Sarnak. Sector estimates for Hyperbolic isometries. GAFA 1175-1200, 2010.

[8] B. H. Bowditch. Geometrical finiteness for hyperbolic groups. J. Funct. Anal., 113(2):245-317, 1993.

[9] H. S. M. Coxeter. The problem of Apollonius. Amer. Math. Monthly, 75:5-15, 1968.

[10] F. Dal'bo. Topologie du feuilletage stable. Ann. Inst. Fourier (Grenoble), 50(3):981-993, 2000.

[11] Diximer. Sur les representations de certains groupes orthonaux C.R. Acad. Sc. Paris, Vol 89, (1960), pp. 3263-3265

[12] W. Duke, Z. Rudnick, and P. Sarnak. Density of integer points on affine homogeneous varieties. Duke Math. J., 71(1):143-179, 1993.

[13] Alex Eskin and C. T. McMullen. Mixing, counting, and equidistribution in Lie groups. Duke Math. J., 71(1):181-209, 1993.

[14] Alex Gorodnik, Hee Oh and Nimish Shah. Strong wavefront lemma and counting lattice points in sectors. Israel J. Math, 176:419-444, 2010. 
[15] Ronald L. Graham, Jeffrey C. Lagarias, Colin L. Mallows, Allan R. Wilks, and Catherine H. Yan. Apollonian circle packings: geometry and group theory. I. The Apollonian group. Discrete Comput. Geom., 34(4):547-585, 2005.

[16] Ronald L. Graham, Jeffrey C. Lagarias, Colin L. Mallows, Allan R. Wilks, and Catherine H. Yan. Apollonian circle packings: number theory. J. of Number Theory, vol 100 (1), pp. 1-45, 2003.

[17] I. S. Gradshteyn and I. M. Ryzhik. Table of integrals, Series and Products Academic press, 2007.

[18] Inkang Kim. Counting, Mixing and Equidistribution of horospheres in geometrically finite rank one locally symmetric manifolds Preprint, arXive:1103.5003

[19] Anthony W. Knapp. Representation Theory of Semisimple Groups. Princeton University Press, 1986.

[20] Alex Kontorovich and Hee Oh. Apollonian circle packings and closed horospheres on hyperbolic 3-manifolds. J. Amer. Math. Soc., 603-648, 2011.

[21] Alex Kontorovich and Hee Oh. Almost prime Pythagorean triples in thin orbits. J. Reiner Angew. Math., Vol 667, 89-131 (2012)

[22] Peter D. Lax and Ralph S. Phillips. The asymptotic distribution of lattice points in Euclidean and non-Euclidean spaces. J. Funct. Anal., 46(3):280-350, 1982.

[23] Gregory Margulis. On some aspects of the theory of Anosov systems. Springer Monographs in Mathematics. Springer-Verlag, Berlin, 2004. With a survey by Richard Sharp: Periodic orbits of hyperbolic flows, Translated from the Russian by Valentina Vladimirovna Szulikowska.

[24] C. T. McMullen. Hausdorff dimension and conformal dynamics. III. Computation of dimension. Amer. J. Math., 120(4):691-721, 1998.

[25] Hee Oh. Dynamics on Geometrically finite hyperbolic manifolds with applications to Apollonian circle packings and beyond. Proc. of ICM (Hyperabad, 2010).

[26] Hee Oh and Nimish Shah. Equidistribution and counting for orbits of geometrically finite hyperbolic groups. Preprint. arXive:1001.2096.

[27] Hee Oh and Nimish Shah. The asymptotic distribution of circles in the orbits of Kleinian groups. Inventiones, Vol 187, 1-35, 2012

[28] Hee Oh. Apollonian packings, Conformal metrics and Hausdorff measures. Preprint.

[29] S.J. Patterson. The limit set of a Fuchsian group. Acta Mathematica, 136:241273, 1976.

[30] Thomas Roblin. Ergodicité et équidistribution en courbure négative. Mém. Soc. Math. Fr. (N.S.), (95):vi+96, 2003.

[31] Thomas Roblin. Sur la théorie ergodique des groupes discrets en géométrie hyperbolique Thése. Université d'Orsay.

[32] Peter Sarnak. Integral Apollonian packings. Amer. Math. Monthly. 118 (2011), 291-306

[33] Peter Sarnak. Letter to J. Lagarias, 2007. available at www.math.princeton.edu/sarnak.

[34] Peter Sarnak. Asymptotic behavior of periodic orbits of the horocycle flow and Eisenstein series. Comm. Pure Appl. Math., 34(6):719-739, 1981.

[35] M. Sugiura. Unitary representations and Harmonic analysis-an introduction Wiley, 1975 
[36] Dennis Sullivan. The density at infinity of a discrete group of hyperbolic motions. Inst. Hautes Études Sci. Publ. Math., (50):171-202, 1979.

[37] Dennis Sullivan. Entropy, Hausdorff measures old and new, and limit sets of geometrically finite Kleinian groups. Acta Math., 153(3-4):259-277, 1984.

[38] Ilya Vinogradov. Effective bisector estimate with applications to Apollonian circle packings. Preprint, 2012

[39] Garth Warner. Harmonic Analysis on semisimple Lie groups. Mém. Soc. Math. Fr. (N.S.), (95):vi+96, 2003.

Mathematics department, Brown university, Providence, Ri

E-mail address: minlee@math.brown.edu

Mathematics department, Brown university, Providence, Ri and KoRea Institute for Advanced Study, Seoul, Korea

E-mail address: heeoh@math.brown.edu 